\author{
MITSUBISHI ELECTRIC RESEARCH LABORATORIES \\ http://www.merl.com
}

\title{
A Synchronization Design for UWB-Based Wireless Multimedia Systems
}

\author{
Zhenzhen Ye, Chunjie Duan, Philip Orlik, Jinyun Zhang, Alhussein Abouzeid
}

TR2010-062 June 2010

\begin{abstract}
Multi-band orthogonal frequency-division multiplexing (MB-OFDM) ultra-wideband (UWB) technology offers large throughput, low latency and has been adopted in wireless audio/video (AV) network products. The complexity and power comsumption, however, are still major hurdles for the technology to be widely adopted. In this paper, we propose a unified synchronizer design targeted for MB-OFDM transceiver that achieves high performance with low implementation complexity. The key component of the proposed synchronizer is a parallel auto-correlator structure in which multiple ACF units are instantiated and their outputs are shared by functional blocks in the synchronizer, including preamble signal detection, time-frequency code identification, symbol timing, carrier frequency offset estimation and frame synchronization. This common structure not only reduces the hardware cost but also minimizes the number of operations in the functional blocks in the synchronizer as the results of a large portion of computation can be shared among different functional blocks. To mitigate the effect of narrowband interference (NBI) on UWB systems, we also propose a low-complexity ACF-based frequency detector to facilitate the design of (adaptive) notch filter in analog/digital domain. The theoretical analysis and simulation show that the performance of the proposed design is close to optimal, while complexity is significantly reduced compared to existing work.
\end{abstract}

\section{IEEE Transactions on Broadcasting}

This work may not be copied or reproduced in whole or in part for any commercial purpose. Permission to copy in whole or in part without payment of fee is granted for nonprofit educational and research purposes provided that all such whole or partial copies include the following: a notice that such copying is by permission of Mitsubishi Electric Research Laboratories, Inc.; an acknowledgment of the authors and individual contributions to the work; and all applicable portions of the copyright notice. Copying, reproduction, or republishing for any other purpose shall require a license with payment of fee to Mitsubishi Electric Research Laboratories, Inc. All rights reserved. 



\title{
A Synchronization Design for UWB-Based Wireless Multimedia Systems
}

\author{
Zhenzhen Ye, Member, IEEE, Chunjie Duan, Member, IEEE, Philip V. Orlik, Member, IEEE, \\ Jinyun Zhang, Fellow, IEEE, and Alhussein A. Abouzeid, Member, IEEE
}

\begin{abstract}
Multi-band orthogonal frequency-division multiplexing (MB-OFDM) ultra-wideband (UWB) technology offers large throughput, low latency and has been adopted in wireless audio/video (AV) network products. The complexity and power consumption, however, are still major hurdles for the technology to be widely adopted. In this paper, we propose a unified synchronizer design targeted for MB-OFDM transceiver that achieves high performance with low implementation complexity. The key component of the proposed synchronizer is a parallel auto-correlator structure in which multiple ACF units are instantiated and their outputs are shared by functional blocks in the synchronizer, including preamble signal detection, time-frequency code identification, symbol timing, carrier frequency offset estimation and frame synchronization. This common structure not only reduces the hardware cost but also minimizes the number of operations in the functional blocks in the synchronizer as the results of a large portion of computation can be shared among different functional blocks. To mitigate the effect of narrowband interference (NBI) on UWB systems, we also propose a low-complexity ACF-based frequency detector to facilitate the design of (adaptive) notch filter in analog/digital domain. The theoretical analysis and simulation show that the performance of the proposed design is close to optimal, while the complexity is significantly reduced compared to existing work.
\end{abstract}

Index Terms-Multi-band orthogonal frequency-division multiplexing (MB-OFDM), narrow-band interference (NBI), synchronization, ultra-wideband (UWB).

\section{INTRODUCTION}

W IRELESS audio/video (AV) systems are gaining popularity as they manage multiple devices throughout a home or office network wirelessly and consequently reduce the clutter and installation cost. Today's AV networks demand high throughput, low latency to support the delivery of high bandwidth content from multiple sources such as high definition video stream and interactive gaming. Traditional narrowband wireless systems are unable to meet these performance require-

Manuscript received January 19, 2009; revised January 10, 2010. First published March 04, 2010; current version published May 26, 2010. This work is funded by Renesas Technology Corp. A preliminary version of parts of this work appears in Proceedings of IEEE ICC 2008.

Z. Ye is with iBasis, Inc., Burlington, MA 01803 USA (e-mail: zhenzhen. ye@ieee.org).

C. Duan, P. Orlik, and J. Zhang are with Mitsubishi Electric Research Labs, Cambridge, MA 02139 USA (e-mail: duan@merl.com; porlik@merl.com; jzhang@merl.com)

A. A. Abouzeid is with Department of Electrical, Computer and Systems Engineering, Rensselaer Polytechnic Institute, Troy, NY 12180 USA (e-mail: abouzeid@ecse.rpi.edu).

Color versions of one or more of the figures in this paper are available online at http://ieeexplore.ieee.org.

Digital Object Identifier 10.1109/TBC.2010.2042499

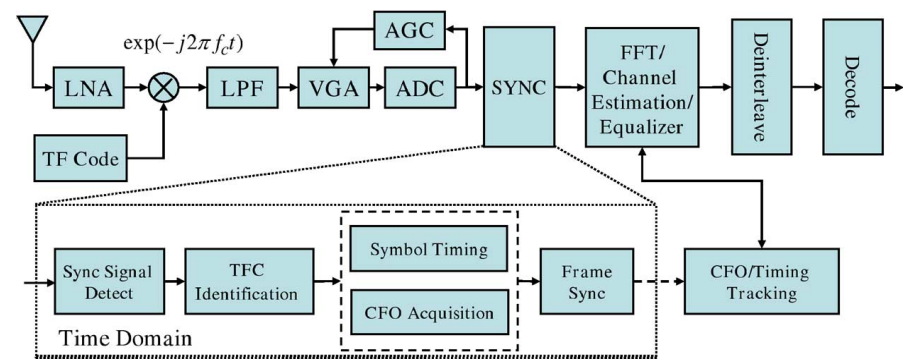

Fig. 1. The synchronization block in a typical MB-OFDM UWB receiver.

ments. Multi-band orthogonal frequency-division multiplexing (MB-OFDM) Ultra-wideband (UWB) technology offers high spectrum efficiency, robustness and outstanding performance under diverse environments and has been employed in some wireless AV products. WiMedia, an open industry association, has published a series of standards and its PHY/MAC specification has been adopted by ECMA as an international standard [1]. With a supported data rate up to $480 \mathrm{Mbps}$, the MB-OFDM UWB link provides sufficient bandwidth to support multiple stream transmission [2].

The transmitter power limit imposed by FCC Part 15 limits the range and bit error performance of all UWB systems. To alleviate this problem, the MB-OFDM UWB technology employs a time-frequency interleaving technique. In doing so, it also provides frequency diversity when systems operate at $106 \mathrm{Mbps}$ or lower data rate. It divides the entire unlicensed $7500 \mathrm{MHz}$ spectrum $(3.1 \sim 10.6 \mathrm{GHz})$ into fourteen $528 \mathrm{MHz}$ bands. Information is transmitted as OFDM modulated symbols and each OFDM symbol is transmitted on a possibly different band using a pre-defined hopping pattern, the time-frequency code (TFC) [1]. On the receiver side, the frequency-hopped RF signal is first down-converted to the baseband signal, then detected, identified and synchronized (at both symbol and frame levels) by the synchronization circuit (SYNC). The synchronized baseband signal is then demodulated and decoded to recover the transmitted information. A typical MB-OFDM UWB receiver architecture is shown in Fig. 1.

Many targeted applications of MB-OFDM UWB technology, such as wireless home entertainment systems are power and cost sensitive, low-power, low-complexity solutions are essential for the prevalence of this technology. The SYNC has been identified as one of the most power-consuming circuits in the baseband. This is due to its higher active duty cycle than other baseband components. Also the performance of the SYNC has direct implication on the overall system performance as errors introduced by the SYNC (e.g., misses in acquisition, estimation 
error in timing and carrier frequency offset, etc.) degrade the overall system performance rapidly. For example, the achievable symbol error rate (SER) of a general OFDM system under an imperfect synchronization can be characterized with the approach proposed in [3]. Unlike most other baseband blocks in the transceiver, the SYNC carries out signal processing in the time domain and therefore is referred to as a pre-FFT processing unit [4], [5]. The SYNC is a multi-task unit and contains several functional blocks that perform: detection of the arrival of the synchronization symbols; identification of the TFC of the received signal; determination of the start of the FFT window, estimation of the carrier frequency offset (CFO) and data frame synchronization.

Research on the SYNC design has been active for over a decade, either for general (single-band) OFDM transceiver structures [4], [6]-[8], [10]-[12], [14], [16], [20], [24], [27] or for specific communication systems using OFDM modulation, such as DVB-T [5], 802.11a WLAN [19] and MB-OFDM UWB [15], [17], [18], [21]-[23], [25], [26]. MB-OFDM UWB systems introduce frequency hopping, which brings up some unique issues for their SYNC design. For example, the preamble signal detection is largely different from that in single-band OFDM systems and the TFC identification problem is quite unique to MB-OFDM as well. In addition, the complexity issue of SYNC design becomes more critical in a UWB system than that in conventional single-band OFDM systems. Most existing work on MB-OFDM UWB focus on optimizing one or two functional blocks that comprise the SYNC circuitry and assume that the other blocks work perfectly [18], [21]-[23], [25]. For example, Li et al. in [21] designed a CFO estimators based on the best linear unbiased estimation (BLUE) principle. The paper is limited to the assumption that inter-symbol-interference (ISI) is eliminated and TFC is known to the estimator. Yoon and Chong proposed a detection and symbol timing design for MB-OFDM UWB [22]. Their design uses the auto-correlation of the cross-correlation output to determine the optimal timing. The paper assumes no frequency hopping and the method has a high complexity. Yao et al. in [26] proposed a joint design for timing and channel estimation where the channel estimation is used to assist timing adjustment. However, the design is not based on the preamble structure defined by the standard [1]. A more comprehensive design was proposed by Jacobs et al. in [23], which provided a synchronization method consists of signal detection, coarse/fine symbol timing, and CFO estimation. However, the paper did not address the TFC identification, instead, it unrealistically assumed that the TFC is known to the receiver at the synchronization stage. Furthermore, most existing work treat the design/optimization of individual SYNC functional blocks as independent of each other [15], [17], [18], [21], [22], [25], which prohibits the cross-block optimization and makes the design unnecessarily complicated.

To the best of our knowledge, there is no existing work that offers a comprehensive study of the SYNC of the MB-OFDM UWB system as a unified structure. We feel that, before proposing any low-complexity solutions for individual blocks, we should first evaluate the design of the SYNC from the top level. Such an approach allows us to treat the individual blocks as components of the structure instead of independent units. We are thus able to identify the overlap in both functionality and resource among blocks within the SYNC and perform the optimization (for either performance or complexity, or both) at the architecture level. It also allows us to choose designs for individual blocks that are "compatible" with one another. Once the overall architecture is defined, any new design techniques or existing ones compatible to the architecture can then be applied in individual blocks to further improve the overall performance and complexity.

In this work, we present a unified SYNC structure designed following this top-down methodology. It is tailored specifically for the low-complexity implementation of the MB-OFDM UWB system [1]. Our proposed design is based on a parallel auto-correlator structure that is shared by all the functional blocks including signal detection, TFC identification, symbol timing, CFO estimation and frame synchronization. Within this unified SYNC structure, several novel designs are proposed for individual functional blocks such as preamble signal detection, TFC identification, CFO estimation and frame synchronization. The sharing of hardware resource and computational results not only reduces the hardware complexity and cost but also minimizes the overall operations of the SYNC (hence low energy consumption). Both theoretical analysis and simulation show that such an architecture enables a low-cost implementation without compromising performances. In addition, we propose a low-complexity narrowband interference (NBI) frequency detector which facilitates the interference detection and cancellation to mitigate the impact of NBI on the performance of synchronization. Any new or existing hardware-oriented implementation techniques can be applied to the proposed synchronization architecture and individual functional block design to achieve low-power, low-complexity synchronizer implementations in a practical MB-OFDM UWB receiver.

The key contributions of this paper include

- A unified SYNC design using a parallel ACF structure. The proposed parallel ACF unit is specifically designed based on the unique features in the MB-OFDM UWB systems, in particular, the frequency hopping patterns and the preamble structure.

- A preamble signal detector and a TFC identification scheme. The detector uses the outputs of the parallel ACF unit to achieve rapid acquisition and TFC identifications. The detection and identification can be implemented with minimum hardware.

- An iterative CFO estimation algorithm. The CFO estimator takes advantage of the phase separation from different outputs of the parallel ACF. The iterative CFO estimator not only has an extended range and a high accuracy, but also can be implemented with low complexity.

- An NBI frequency detector. The simple ACF-based frequency detector offers good performance and can be used to facilitate an adaptive notch filter design in the analog/ digital domain.

- Theoretical analysis on the performance of individual functional blocks. The analysis provides insights on the achievable performance of the proposed design and has been proven effective by simulation results.

The remainder of the paper is organized as follows. Section II introduces the MB-OFDM UWB signal model and the NBI signal model. Section III outlines the difference of auto-cor- 
relation function and cross-correlation function in computation cost and implementation complexity. In Section IV, we present the overall architecture of the proposed SYNC, followed by the details of the design and the operation of individual functional blocks. The proposed NBI frequency detector is also discussed in this section. The performance analysis and simulation results are presented in Section V. Section VI draws the conclusion.

\section{Signal Model}

\section{A. An MB-OFDM Signal Model}

In [1], a transmitted symbol is defined as an OFDM symbol with $N=128$ sub-carriers followed by $N_{g}=32$ samples of zero-padding. An additional $\left(N_{g}^{\prime}-N_{g}\right) T=5$ sample periods are inserted for RF band switching where the sampling interval $T \sim 2$ ns. The resulting OFDM symbols are of total length $T_{s}=\left(N+N_{g}^{\prime}\right) T$, which includes $N_{s}=N+N_{g}^{\prime}=165$ samples. Each OFDM symbol contains $Q<N$ data symbols $a_{l, k}$, where $l$ denotes the OFDM symbol time index and $k \in$ $[-Q / 2,-1] \cup[1, Q / 2]$ denotes the subcarrier frequency index ${ }^{1}$, the transmitted baseband signal is given by ${ }^{2}$

$$
s(t)=\frac{1}{\sqrt{N T}} \sum_{l} \sum_{k} a_{l, k} e^{j 2 \pi(k / N)\left(t-l T_{s}\right) / T} u\left(t-l T_{s}\right)
$$

where $u(t)=1,0 \leq t<N T$ and zero otherwise.

The equivalent baseband frequency selective fading channel including the actual UWB channel impulse response (CIR) at the corresponding frequency band as well as the effect of analog path of the transceiver can be modeled as

$$
h(\tau)=\sum_{i} h_{i} \delta\left(\tau-\tau_{i}\right)
$$

where the complex path gains $\left\{h_{i}\right\}$ and the path delays $\left\{\tau_{i}\right\}$ are assumed to be time-invariant within one data frame [1]. The maximum path delay is $\tau_{\max }$. Taking into account the CFO between the transmitter and the receiver, the discrete received baseband signal is given by

$$
r[m]= \begin{cases}e^{j 2 \pi m \Delta f T} \sum_{i} h_{i} s\left(m T-\tau_{i}\right)+\nu[m], & f_{t}=f_{r} \\ 0 . & f_{t} \neq f_{r}\end{cases}
$$

where $\nu[m]$ is the complex zero-mean white Gaussian noise with the variance $\sigma_{\nu}^{2}, f_{t}$ is the center frequency of the transmitting RF band, $f_{r}$ the center frequency of the receiver RF band and $\Delta f$ is the frequency offset. For channel estimation and data demodulation, the receiver performs the overlap-and-add (OLA) operation on the received symbols and then demodulates the data via FFT. When a symbol timing bias $d$ is taken into account ${ }^{3}$, the $l$ th OFDM symbol for FFT operation is given by $\mathbf{r}_{l}=\left[r_{l, 0}+r_{l, N}, r_{l, 1}+r_{l, N+1}, \ldots, r_{l, N_{g}-1}+\right.$

\footnotetext{
${ }^{1}$ The DC component in OFDM symbols is set to 0 .

${ }^{2}$ Note that (1) only models the OFDM symbols on one frequency band. Since the transmissions/receptions on three bands are independent and symmetric, this model is valid as long as our concern is the reception of an individual OFDM symbol.

${ }^{3}$ The imperfection of the baseband sampling clock also has effect on the system behavior, however, it is insignificant during the preamble period [28].
} From here on, we assume a perfect sampling clock in synchronization. $\left.r_{l, N+N_{g}-1}, r_{l, N_{g}}, \ldots, r_{l, N-1}\right]$ where $r_{l, m}=r\left[m+d+l N_{s}\right]$ $m=0,1, \ldots, N-1$.

\section{B. A Narrowband Interference (NBI) Signal Model}

As MB-OFDM UWB occupies a broad frequency band, the coexistence with other existing or future narrowband systems is an important issue for MB-OFDM UWB system design. The narrowband interference (NBI) has been shown to have a big impact on the performance of OFDM systems [7], [15]. For synchronization operations, NBI increases the miss/false detection probabilities and degrades the accuracy of symbol timing and CFO estimation.

To model the effect of NBI on a specific sub-band of the MB-OFDM signal, we adopt a linearly modulated NBI signal model in [15]

$$
I(t)=A_{i} \sum_{n} b_{n} e^{j\left(2 \pi f_{i} t+\phi_{i}\right)} p\left(t-n T_{i}\right)
$$

where $A_{i}, f_{i}$ and $\phi_{i}$ represent the carrier amplitude, central frequency and phase of the NBI signal respectively, with respect to (w. r. t.) the considered sub-band of the MB-OFDM signal. $p(t)$ is a rectangular pulse. Here we assume that the OFDM signal on the sub-band has been down-converted to the baseband and the only in-band NBI is considered, i.e., $f_{i} \in[-(1 / 2 T),(1 / 2 T)]$. The sequence $\left\{b_{n}\right\}$ are the modulated symbols of the NBI signal and $T_{i}$ is a symbol duration of the modulated NBI signal. If $T_{i} \sim N T$, the NBI signal might affect several subcarriers of the MB-OFDM UWB system. If $T_{i} \gg N T$, the NBI signal can be regarded as an unmodulated tone centered at the frequency $f_{i}$.

\section{DeSIGN COMPLEXITY CONSIDERATION}

Before looking at any specific design, we would like to first identify two basic operations widely used in OFDM synchronization design for detecting repeated, pre-defined signals:

1) cross-correlation function (CCF): $C C(d)=\sum_{m} r^{*}[m+$ $d] s[m]$, in which the received signal $\{r[m]\}$ is correlated with a known synchronization symbol $\{s[m]\}$;

2) auto-correlation function (ACF): $A C(d)=\sum_{m} r^{*}[m+$ $d] r[m+M+d]$, in which the correlation is carried out between the segments of the received signals with different delays, i.e., $\{r[m]\}$ and $\{r[m+M]\}$.

Even though two functions look similar, there is a significant difference in computation complexity between them. The ACF can be rewritten into an iterative structure as follows

$$
\begin{aligned}
A C(d) & =\sum_{m=1}^{K} r^{*}[m+d] r[m+M+d] \\
& =A C(d-1)+U[K+d]-U[d]
\end{aligned}
$$

where $U[d] \triangleq r^{*}[d] r[M+d]$. Therefore, the ACF only requires 1 complex multiplication (for calculating $U[K+d]$ ) and 2 complex additions for every new sample. It can be readily implemented in hardware using one complex multiplier, two adders and some delay elements (e.g., memory). On the other hand, no such efficient implementation is available for CCF. In the case of MB-OFDM UWB synchronization symbol $\{s[m]\}$ with the 


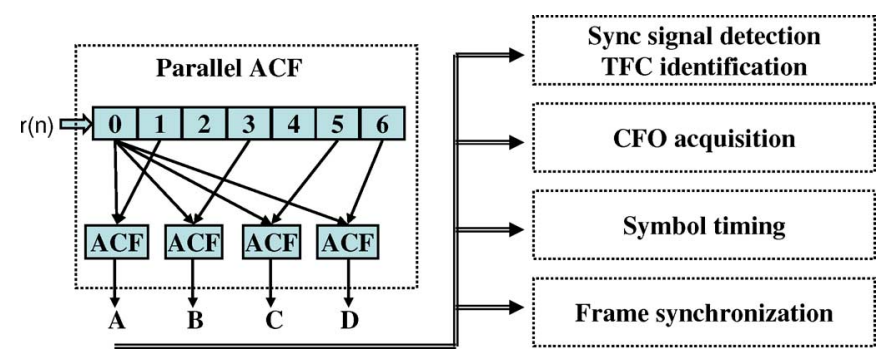

Fig. 2. A top-level structure of the proposed parallel ACF-based synchronizer. Numbers in delay blocks indicate synchronization symbol delay, i.e., a delay of 165 samples.

length of 128 samples, CCF requires 256 real multiplications ${ }^{4}$ and 127 complex additions per sample, much more expensive than an ACF-based design.

For pure complexity and power efficiency, an ACF-based SYNC is definitely appealing. The concern, however, is how well can a pure ACF-based design perform. In the rest of this paper, we show that a well-designed ACF-based SYNC does not compromise the system performance. On the contrary, the robustness to frequency offsets makes it more attractive than the CCF-based design in signal detection, TFC identification, symbol timing and frame synchronization.

\section{AN ACF-BASED SYNCHRONIZER DESIGN}

\section{A. The Overall SYNC Structure}

We propose an ACF-based SYNC structure as shown in Fig. 2. The design is not only motivated by the complexity issue discussed above, but also based on two unique characteristics of WiMedia's MB-OFDM UWB signal design: (i) the time-frequency hopping pattern of MB-OFDM UWB signal and (ii) the structure of the preamble of a data packet defined in [1]. The preamble of a data packet consists of 24 repeated synchronization symbols. The symbols are 128-point time-domain real pseudo-random (PR) sequences, unique to given TFCs. The symbols do not undergo any OFDM modulation. The 128-point sequence is then zero padded with 37 zero samples so that a single symbol is the same length as an OFDM symbol (165 samples). The repeated synchronization symbols allow the ACF to be used in design. The TFC patterns defined in [1] motivate us to adopt a parallel ACF structure. As we would show later, the proposed parallel ACF structure not only allows the receiver to achieve rapid signal acquisition and TFC identification, but also facilitates the design of an iterative $\mathrm{CFO}$ estimator.

In the parallel ACF block, there are four ACF units that performs auto-correlation between the input signal and signals that are delayed by 1, 3, 5 and 6 symbols length. The outputs of the ACF blocks can be expressed as

$$
A C(d p ; W) \triangleq \sum_{m=0}^{N+W-1} r^{*}[m+d] r\left[m+p N_{s}+d\right]
$$

where $p$ has the values of $1,3,5,6$ respectively, $W$ is the number of samples in each segment in addition to $N$, the number of samples in a FFT operation. $N+W$ is therefore the length of the

\footnotetext{
${ }^{4}$ This is equivalent to at least 64 complex multiplications.
}

TABLE I

Output Patterns of the ACF-Based Parallel Signal Detector, After COMPARING TO A GIVEN THRESHOLD

\begin{tabular}{l||c|c|c|c}
\hline TFC group & A & B & C & D \\
\hline \hline TFC 1, 2 & 0 & 1 & 0 & 1 \\
\hline \multirow{2}{*}{ TFC 3, 4 } & 1 & 0 & 0 & 1 \\
& 0 & 0 & 1 & 1 \\
\hline TFC 5-7 & 1 & 1 & 1 & 1 \\
\hline
\end{tabular}

received signal segments that are processed with auto-correlation operations. The value of $W$ is chosen within $\left[0, N_{g}\right]$. The hardware to implement the parallel ACF block involves 4 complex multipliers, 8 adders and delay elements. Note that this is only a small fraction of a single 128-point CCF implementation.

During the synchronization, each ACF unit outputs one value in every clock cycle at $528 \mathrm{MHz}$. The outputs are fed into all functional blocks of the SYNC. The following sections describe the operation details of individual blocks.

\section{B. Signal Detection and TFC Identification}

Before the synchronization is acquired and TFC identified, a receiver needs to scan through all sub-bands, i.e., it stays on one band and "listens" to possible incoming preamble signals for a period of time. If no packet is detected, the receiver switches to a different band and continue listening. For any incoming packet, because of the frequency hopping, only the symbols in the sub-band that the receiver is listening can be "heard". As an example, for TFC 1 , the receiver listening on sub-band 1 receives only one of every three preamble symbols. With the proposed parallel ACF structure, the detection of signal is declared immediately once the ACF outputs (after comparing to a given threshold) matches the ones given in Table I. Note that the minimum Hamming distance of different output patterns in Table I is two and thus a single error in the output pattern can be detected. We will see that the output $\mathbf{D}$ in Fig. 2 not only improves the robustness of the detection but also is needed in refining $\mathrm{CFO}$ estimation.

As shown in Table I, the detector output pattern also indicates the TFC group of the detected signal. TFC 5, 6 and 7 are implied by the RF subband, no further process is needed. If the received signal belongs to TFC $1-4$, additional steps are taken to determine the TFC. There are two approaches the receiver can use to determine the transmitted TFC:

1) perform $\mathrm{CCF}$ between the received signal segment and one of the known synchronization symbols in the group of the TFCs; the TFC is determined by comparing the CCF value with a pre-defined threshold; or

2) switch to a different sub-band and continue to perform ACF operations on the incoming baseband signal and determine the TFC by identifying the location of the ACF output peaks.

The first approach requires additional hardware and is more computationally intensive. In addition, even though the different PR sequences of synchronization symbols have a good auto-correlation property, their cross-correlation property is fairly poor. This results in a low peak to average ratio at the CCF output, which makes it difficult to set the threshold and the design is sensitive to noise. In contrast, the second does not require an 


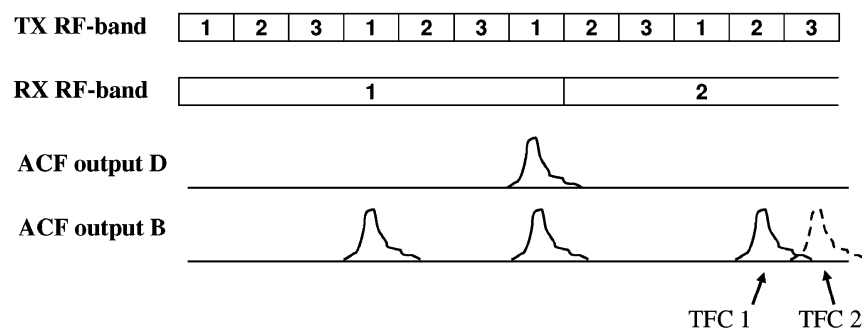

Fig. 3. An illustration on the ACF-based TFC identification via bandswitching. The output pattern of the signal detector is assumed to be $\left[\begin{array}{llll}0 & 1 & 0 & 1\end{array}\right]$ ( $B=1, D=1)$, i.e., the possible TFC is either 1 or 2 . The ACF peak position uniquely determines the TFC of the received signal.

absolute threshold. It is also computationally cheaper and, requires little additional hardware resources as the existing hardware (i.e., ACF units) are reused. The procedure is illustrated in Fig. 3. Assuming the output pattern $\left[\begin{array}{llll}0 & 1 & 0 & 1\end{array}\right]$ is detected on sub-band 1, the receiver switches to sub-band 2 and continues to do ACF operations on two signal segments with the separation of $3 N_{s} T$. The peak position of the ACF outputs implies the TFC of the received signal.

\section{OFDM Symbol Timing}

After the preamble signal is detected and its TFC identified, the SYNC needs to search for the start of an OFDM symbol. This step is referred as the symbol timing. An inaccurate timing not only introduces inter-carrier-interference (ICI) and (possible) ISI, but also affects the quality of channel estimation and the total signal energy collected in the FFT window. It therefore has significant implication on bit-error-rate (BER) performance.

An optimal symbol timing point should maximize the signal-to-noise ratio (SNR) at the FFT output. For illustration, consider the MB-OFDM UWB signal of TFC 1 or 2 , due to the large separation between received symbols on the same sub-band, ISI due to timing error is negligible ${ }^{5}$, the optimal timing point $d_{o p t}$ is defined as [28]

$$
d_{\text {opt }}=\arg \max _{d}\left\{\frac{\sigma_{s}^{2}}{\sigma_{I C I}^{2}+\left(1+\frac{N_{g}}{N}\right) \sigma_{\nu}^{2}}\right\}
$$

where $d$ is the timing bias (in the unit of sampling period, $T$ ), w. r. t. the start of a received OFDM symbol, $\sigma_{s}^{2}$ is the signal power and $\sigma_{I C I}^{2}$ is the ICI power after FFT operation. Specifically, we can show that [28]

$$
\begin{aligned}
\sigma_{s}^{2}= & \int_{0}^{\eta} E\left[|h(\tau)|^{2}\right]\left(1-\frac{\eta-\tau}{N T}\right)^{2} d \tau+\int_{\eta}^{\eta+T_{g}} E\left[|h(\tau)|^{2}\right] d \tau \\
& +\int_{\eta+T_{g}}^{\tau_{\max }} E\left[|h(\tau)|^{2}\right]\left(1-\frac{\tau-\eta-T_{g}}{N T}\right)^{2} d \tau
\end{aligned}
$$

and

\footnotetext{
${ }^{5}$ The analysis on TFC 3-7 where ISI is non-negligible can be manipulated in
} exactly the same way in [28] with a slightly more tedious derivation.

$$
\begin{aligned}
\sigma_{I C I}^{2}= & \int_{0}^{\eta} E\left[|h(\tau)|^{2}\right]\left(1-\frac{\eta-\tau}{N T}\right)\left(\frac{\eta-\tau}{N T}\right) d \tau \\
& +\int_{\eta+T_{g}}^{\tau_{\max }} E\left[|h(\tau)|^{2}\right]\left(1-\frac{\tau-\eta-T_{g}}{N T}\right)\left(\frac{\tau-\eta-T_{g}}{N T}\right) d \tau
\end{aligned}
$$

where $T_{g} \triangleq N_{g} T$ and $\eta \triangleq d T$, i.e., the timing bias in continuous time domain. A close-formed solution to (6) is difficult to obtain. Alternatively, we look at a slightly different optimization criterion which maximizes the difference between the signal power and the sum of ICI and noise power, i.e.,

$$
\hat{d}_{o p t}=\arg \max _{d}\left\{\sigma_{s}^{2}-\sigma_{I C I}^{2}-\left(1+\frac{N_{g}}{N}\right) \sigma_{\nu}^{2}\right\} .
$$

It can be shown that [28]

$$
\int_{0}^{\hat{d}_{o p t} T} E\left[|h(\tau)|^{2}\right] d \tau \approx \int_{\hat{d}_{o p t} T+T_{g}}^{\tau_{\max }} E\left[|h(\tau)|^{2}\right] d \tau .
$$

(8) can be interpreted as the optimal timing point $\hat{d}_{\text {opt }}$ approximately equalizes channel energy in $\left[0, \hat{d}_{o p t} T\right]$ and $\left[\hat{d}_{\text {opt }} T+T_{g}, \tau_{\text {max }}\right]$. We emphasize that (8) holds for any channel spread $\tau_{\max }$. Note that if $\tau_{\max } \leq T_{g}$, the channel energy out of $\left[d T, d T+T_{g}\right]$ is zero when $d$ is optimal and thus (8) is trivially satisfied.

In practice, the symbol timing schemes for OFDM systems generally can be classified as CCF-based [17], [19], ACF-based [5], [11], [12] and hybrid [15], [20]. Both the hybrid and CCFbased metrics are computationally intensive and the CCF-based ones are also sensitive to frequency offset, though the hybrid ones are expected to have the best performance and resilience to narrowband interference [15], [20]. Numerous ACF-based timing schemes have been proposed in the literature [5], [11], [12], [14]. All these schemes can be implemented with lowcomplexity. The major difference among the schemes is in normalizing/biasing the auto-correlation values to meet the different criteria [14]. Here we use the maximum correlation (MC) metric in the proposed synchronizer, i.e.,

$$
\hat{d}_{1}=\arg \max _{d}\left\{\left|A C\left(d ; p, N_{g}\right)\right|\right\} .
$$

Based on the derivation given in Appendix A, we find that the metric (9) satisfies

$$
\int_{0}^{\hat{d}_{1} T}|h(\tau)|^{2} d \tau \approx \int_{\hat{d}_{1} T+T_{g}}^{\tau_{\max }}|h(\tau)|^{2} d \tau
$$

that is, the timing point $\hat{d}_{1}$ from the metric in (9) approximately equalizes the instant channel energy in the window $\left[0, \hat{d}_{1} T\right]$ and $\left[\hat{d}_{1} T+T_{g}, \tau_{\text {max }}\right]$. Compared to (8), we find that $\hat{d}_{1}$ (approximately) maximizes the criterion (7) for a given channel realization $\{h(\tau)\}$. Thus the metric given in (9) can achieve a near-optimal performance. 


\section{Carrier Frequency Offset (CFO) Estimation}

The SYNC is responsible for estimating the carrier frequency offset (CFO), so the CFO can be compensated before the receiver starts channel estimation and data demodulation. CFO estimation is typically done in two stages: pre-FFT and post-FFT. The pre-FFT operation gives the initial CFO estimation, which is critical as it affects the following channel estimation and data demodulation, and is typically implemented as part of synchronization. Once the system is synchronized, the residual CFO can be removed via pilot-tone tracking in the frequency domain during the data demodulation, the so called post-FFT CFO tracking [5].

In the proposed SYNC design, the CFO estimation is carried out in parallel with symbol timing. In MB-OFDM UWB, we note that the inter-subcarrier spacing is $4.125 \mathrm{MHz}$ and the minimum interval between two repeated preamble symbols on the same band in all TFCs would not exceed $3 N_{s} T$. On the other hand, the CFO w. r. t. the absolute central frequency is specified to be within 6 \pm 20 ppm [1] and therefore, the maximum relative CFO $\Delta f$ between two nodes is $\pm 40 \mathrm{ppm}$ (This translates into $\sim 160 \mathrm{kHz}$ for a carrier center frequency of 4 $\mathrm{GHz}$ ). Thus, the maximum phase difference between two repeated preamble symbols on the same band would not exceed $2 \pi \times 3 N_{s} \Delta f T \leq 0.3 \pi$ in the first band group. In fact, for all band groups 1-5 defined in [1], the maximum CFO would not exceed 1/2 inter-subcarrier spacing, which implies that the estimation of CFO based on the phase difference between two nearest synchronization symbols on the same band in the time domain will not have any phase ambiguity and is sufficient to correct a major portion of the actual CFO.

The basic operation for CFO estimation in the time domain is to estimate the phases of the $\mathrm{ACF}$ values close to the maximum value of $\left|A C\left(d ; p, N_{g}\right)\right|$ [5], [11], [17]. Since the value of $p$ in $\left|A C\left(d ; p, N_{g}\right)\right|$ indicates the delay interval between two correlated symbols, we observe that:

- a small $p$ allows us to estimate a large range of CFO without phase ambiguity since the phase of an ACF value $A C\left(d ; p, N_{g}\right)$ is given by $p 2 \pi N_{s} \Delta f T$;

- a large $p$ can yield a high frequency resolution given that there is no phase ambiguity.

To satisfy both range and accuracy requirements, we do need to use the ACF outputs with multiple different values of $p$, and some of them are already available from the parallel ACF block in the signal detection and TFC identification stage (i.e., the outputs A, B, C and D in Fig. 2).

We propose an iterative CFO estimation algorithm to take advantage of different values of $p$ to improve both the range and the accuracy of the estimation (See Fig. 4). We emphasize that the iterative $\mathrm{CFO}$ estimation structure is fairly general and includes the traditional ACF-based non-iterative CFO estimators in [5], [11], [17] as a special case of the iteration number set as one. The details of the algorithm are given in Table II, where $\Gamma_{k}$ is the set of timing points close to the peak of $\left|A C\left(d ; p_{k}, N_{g}\right)\right|$ and $\alpha \in(0,1]$ is the stepsize for updating the estimation in each iteration. The value of $\alpha$ is usually chosen to be one or close to one unless the SNR is very low (e.g., less than $-5 \mathrm{~dB}$ ). Initially, the ACF values with the smallest value of $p$ (e.g., $p=3$ in

\footnotetext{
${ }^{6} 1 \mathrm{ppm}=10^{-6}$ of the reference frequency.
}

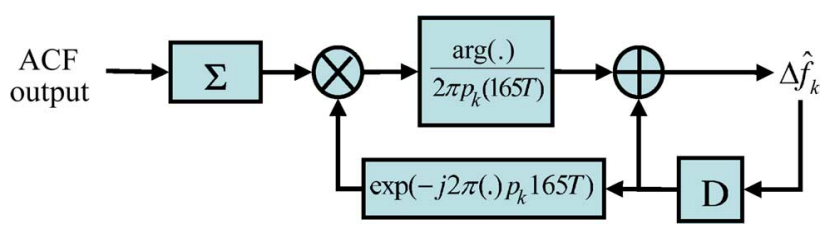

Fig. 4. Structure of the iterative CFO estimator, where $N_{s}=165$ and $\alpha=1$.

TABLE II

ITERATIVE CFO ESTIMATION

\begin{tabular}{l}
\hline \hline (1) Known: $A C\left(d ; p_{1}, N_{g}\right), d \in \Gamma_{1}, p_{1} \geq 1$ \\
(1a) $S_{1, \Gamma_{1}}=\sum_{d \in \Gamma_{1}} A C\left(d ; p_{1}, N_{g}\right) ;$ \\
(1b) $\Delta \hat{f}_{1}=\frac{1}{2 \pi p_{1} N_{s} T} \arg \left\{S_{1, \Gamma_{1}}\right\} ;$ \\
(2) For $k \geq 2, \operatorname{known} A C\left(d ; p_{k}, N_{g}\right), d \in \Gamma_{k}, p_{k}>p_{k-1}$ \\
(2a) $S_{k, \Gamma_{k}}=\sum_{d \in \Gamma_{k}} A C\left(d ; p_{k}, N_{g}\right) e^{-j 2 \pi \Delta \hat{f}_{k-1} p_{k} N_{s} T}$; \\
(2b) $\delta \hat{f}_{k}=\frac{1}{2 \pi p_{k} N_{s} T} \arg \left\{S_{k, \Gamma_{k}}\right\} ;$ \\
(2c) $\Delta \hat{f}_{k}=\Delta \hat{f}_{k-1}+\alpha \delta \hat{f}_{k} ;$ \\
(3) Go back to (2) for further refinement on the estimation.
\end{tabular}

TFC 1 or $2, p=1$ in TFC 3-7) is used in estimation (i.e., the step (1) of Table II), which covers the largest CFO range of estimation. Then, in the step (2) of Table II, the estimated CFO is used to correct the phases of the incoming ACF values and the residue $\mathrm{CFO}$ which generally has a much smaller dynamic range than the initial one can be estimated from these ACF values with larger values of $p$ (e.g., the ACF values with $p=6$ at the output $\mathbf{D}$ is already available in the signal detection and TFC identification stage). This refinement procedure can be controlled to balance the performance of estimation and the computation cost. The simulation results in Section V-B shows that two iterations are sufficient to remove most portion of the CFO.

\section{E. Frame Synchronization}

The preamble uses a frame synchronization cover sequence to modulate the polarities of the 24 synchronization symbols [1]. The goal of frame synchronization is to synchronize the receiver to the cover sequence. Generally this process starts when the TFC identification, symbol timing and CFO estimation/correction are completed and the receiver frequency hopping is already enabled.

Our proposed frame synchronization design is again based on the ACF outputs. It is much simpler than the ones using CCF output as since there is no accumulative phase rotation in the ACF outputs. The polarity change in the cover sequence produces a negative peak at the corresponding ACF outputs. For improved robustness, multiple ACFs (with different delays) can be used. For example, the cover sequence for TFC 1 has twenty-one "+1" followed by three " -1 ", as [ . . $1111-1-1-1$, both ACF output $\mathbf{B}$ and $\mathbf{D}$ will have the output as [... $1111-1-$ $1-1]$.

\section{F. Narrowband Interference (NBI) Mitigation}

NBI can significantly degrade the UWB receiver performance. For an ACF-based SYNC design, it may introduce a bias at the ACF outputs and thus result in synchronization errors. Also, typically the receiver RF front end has limited dynamic range. Left unsuppressed, NBI can easily destroy the linearity and jam the front end completely. We propose a 


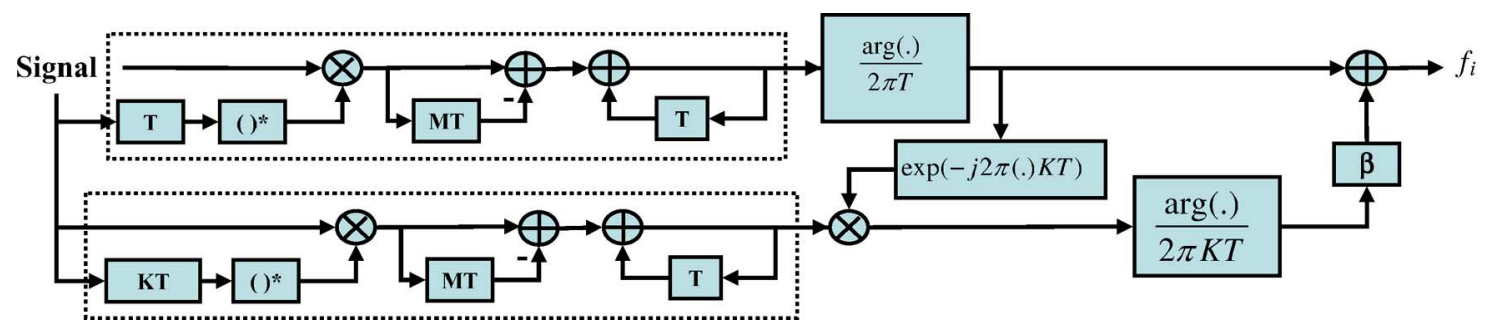

Fig. 5. Structure of a two-stage ACF-based NBI frequency detector. $M$ stands for the number of terms in the summation in (11).

low-complexity NBI frequency detector which is implemented in parallel to the proposed SYNC and targeted to detect the central frequency of the NBI. The detected NBI frequency is used to facilitate the design of a (adaptive) notch filter in analog/digital domain to eliminate the effect of NBI on the synchronization and other signal processing. Different from the existing frequency domain NBI detection schemes [15], the proposed frequency detector operates in time domain before the FFT operation takes place. Such an implementation responses more rapidly to the NBI and also minimizes the impact of NBI in the synchronization stage.

The basic idea of the proposed NBI frequency detector is to acquire and refine the estimation of NBI central frequency from the phases of ACF values, which is similar to the procedure of the proposed iterative CFO estimation. Fig. 5 illustrates the structure of a two-stage frequency detector. It operates independently of the rest of the SYNC blocks. Since the central frequency of NBI can vary in the range of $[-(1 / 2 T),(1 / 2 T)]$, the ACF operation in the first stage of the frequency detector is carried out between samples separated by one sample period. Assume the received NBI signal sampled at $t=m T$ is given by $\tilde{I}[m]=I[m]+\nu[m]$, where $I[m]=A_{i} b[m] e^{j\left(2 \pi f_{i} m T+\phi_{i}\right)}$ and $\nu[m]$ is the noise sample with the variance $\sigma_{\nu}^{2}$. The auto-correlation is given by

$$
\sum_{m} \tilde{I}^{*}[m] \tilde{I}[m+1]=\sum_{m} A_{i}^{2}\left(b^{*}[m] b[m+1]\right) e^{j 2 \pi f_{i} T}+\nu_{i}
$$

where $\nu_{i}=\sum_{m} I^{*}[m] \nu[m+1]+\sum_{m} I[m] \nu^{*}[m+$ $1]+\sum_{m} \nu^{*}[m] \nu[m+1]$ is the composite noise term. Since the symbol duration of NBI signal $T_{i} \gg T$, $b^{*}[m] b[m+1] \approx|b[m]|^{2}$. The NBI frequency estimation at the first stage is thus given by

$$
\hat{f}_{i}=\frac{1}{2 \pi T} \arg \left\{\sum_{m} \tilde{I}^{*}[m] \tilde{I}[m+1]\right\} .
$$

In the second stage, by assuming that the bandwidth of the narrowband signal $B_{i}<1 / K T$, e.g., with $1 / T=528 \mathrm{MHz}$, $K=10$ implies that $B_{i}<52.8 \mathrm{MHz}$ which holds for most of existing narrowband systems, the ACF operation is carried out between the samples separated by $K T$. The corresponding estimation on the residue $f_{i}-\hat{f}_{i}$ is thus given by

$$
\delta \hat{f}_{i}=\frac{1}{2 \pi K T} \arg \left\{\sum_{m} \tilde{I}^{*}[m] \tilde{I}[m+K] e^{-j 2 \pi \hat{f}_{i} K T}\right\},
$$

and the refined estimation on the NBI central frequency is given by $\hat{f}_{i}+\beta \delta \hat{f}_{i}$, where $\beta \in(0,1]$ is a stepsize for updating the estimation of the frequency. The two-stage NBI frequency detector in Fig. 5 can be further generalized to a multiple-stage one. However, as the accuracy requirement on estimating the central frequency of the $\mathrm{NBI}$ is not as stringent as that for $\mathrm{CFO}$, the two-stage NBI detector is adequate given the computation and hardware cost constraints.

\section{The Evaluation of the Proposed ACF-Based SYNCHRONIZER}

In this section, we evaluate the performance of the proposed ACF-based synchronizer with both analytical characterization and simulation validation. In simulations, since there is no such a comprehensive synchronization design for MB-OFDM UWB in the literature as we proposed here, we only compare the performance of individual functional blocks in the proposed design to existing schemes whenever available. The basic system parameters of MB-OFDM UWB follow the ones specified in [1]. We consider the first band group whose central frequencies of its three sub-bands are $\{3.432,3.960,4.488\} \mathrm{GHz}$. For ACF operations, we consider the ACF on two received signal segments with the length $N+N_{g}=160$ and a separation of $p N_{s} T$, $p=1,3,5,6,12$. Two types of UWB channels, CM1 and CM4, are used in the simulation [9].

We also provide a quantitative comparison on the computational complexity between the functional blocks in the proposed design and existing ones.

\section{A. The Evaluation of Signal Detection and TFC Identification}

We first quantify the performance of the proposed signal detector. There are three scenarios be to considered:

1) When synchronization symbols are present in both signal segments, the distribution of the peak (absolute) ACF value $X_{1}$ is given by (24) in Appendix B. Then, the detection probability $P_{d}$ is given by

$$
P_{d}=P_{r}\left[X_{1}>\lambda\right]=Q\left(\frac{A_{d}}{\sqrt{\sigma_{\nu_{d}}^{2} / 2}}, \frac{\lambda}{\sqrt{\sigma_{\nu_{d}}^{2} / 2}}\right),
$$

where $\sigma_{\nu_{d}}^{2}$ is the variance of the composite noise given in (23), $Q(\cdot, \cdot)$ is the generalized Marcum's $Q$ function [13] and $\lambda$ is the detection threshold. In TFC 1-4 where the transmitted signal hops among three bands, when $d$ is close to where the auto-correlation output peaks, $A_{d} /(N+$ $N_{g}$ ) can be seen as the peak signal power (averaged over $N+N_{g}$ samples). The actual received SNR on a band 

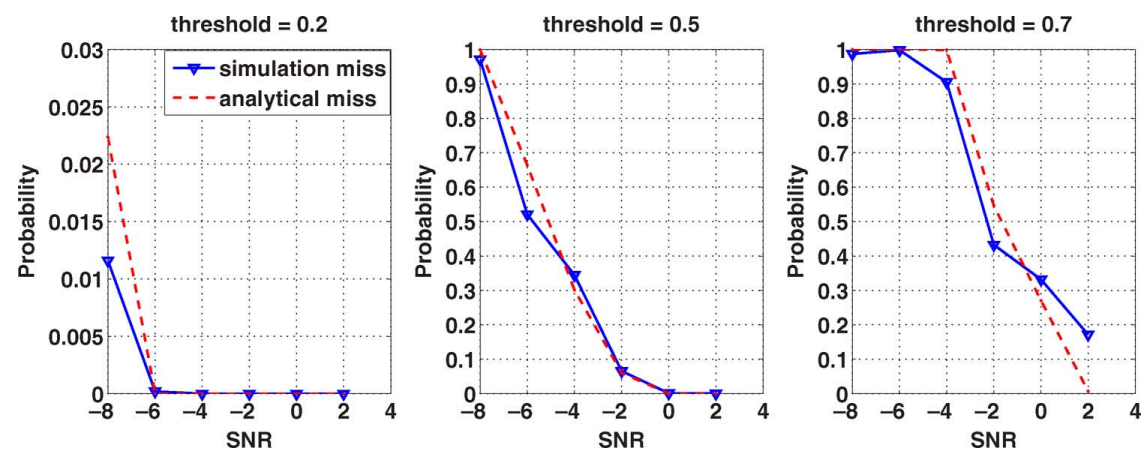

Fig. 6. The comparison of analytical miss detection probabilities $\left(1-\mathcal{P}_{d}\right)$ and the simulation results, with TFC 1 and in UWB CM1 channels.

$S N R_{r} \approx\left(A_{d} / \kappa\left(N+N_{g}\right) \sigma_{\nu}^{2}\right)$ (for a given channel realization), where $\kappa=3$. For TFC 5-7, since all symbols are in the same band, $\kappa=1$. Thus

$$
P_{d} \approx Q\left(\frac{\kappa S N R_{r} \sqrt{2\left(N+N_{g}\right)}}{\sqrt{2 \kappa S N R_{r}+1}}, \frac{\lambda \sqrt{\frac{2}{N+N_{g}}}}{\sigma_{v}^{2} \sqrt{2 \kappa S N R_{r}+1}}\right) .
$$

2) When the synchronization symbol is presents in only one of the two signal segments, using the distribution of the peak (absolute) ACF value $X_{2}$ given by (25) in Appendix B, The false alarm probability $P_{f, 01}$ is

$$
P_{f, 01}=P_{r}\left[X_{2}>\lambda\right] \approx \exp \left[\frac{-1}{\left(N+N_{g}\right)} \frac{\lambda^{2}}{\sigma_{v}^{4}\left(\kappa S N R_{r}+1\right)}\right] \text {. }
$$

3) When no synchronization symbol is present in either signal segment, the distribution of the peak (absolute) ACF value $X_{3}$ is given by (26) in Appendix B. The false alarm probability $P_{f, 00}$ becomes

$$
P_{f, 00}=P_{r}\left[X_{3}>\lambda\right]=\exp \left[-\frac{\lambda^{2}}{\left(N+N_{g}\right) \sigma_{v}^{4}}\right] .
$$

For the proposed parallel signal detector, we can then derive the probability of correct detection and identification of the TFC group when the synchronization symbols are present. Assume all 24 repeated synchronization symbols in a preamble of a data packet are available [1], this probability can be approximated as

$$
\mathcal{P}_{d}=1-\left[1-\left(1-P_{f, 01}\right)^{2} P_{d}^{2}\right]^{6}
$$

for TF code 1-4 where there are 8 synchronization symbols on each frequency sub-band; and

$$
\mathcal{P}_{d}=1-\left[1-4\left(1-P_{d}\right) P_{d}^{3}-P_{d}^{4}\right]^{18}
$$

for TF code 5-7 where all 24 synchronization symbols are in the same band. The presence of the synchronization symbol sequence with TFC 5-7 is declared if at least three of the four outputs (i.e., $[A, B, C, D]$ ) of the parallel signal detector exceed the given threshold. Fig. 6 shows that the analytical missed detection probability (i.e., $1-\mathcal{P}_{d}$ ) matches the simulation results very well.

Similarly, we can characterize the performance of the ACFbased TFC identification with RF band-switching for TFC 1-4. Using the proposed TFC identification procedure (see Fig. 3),

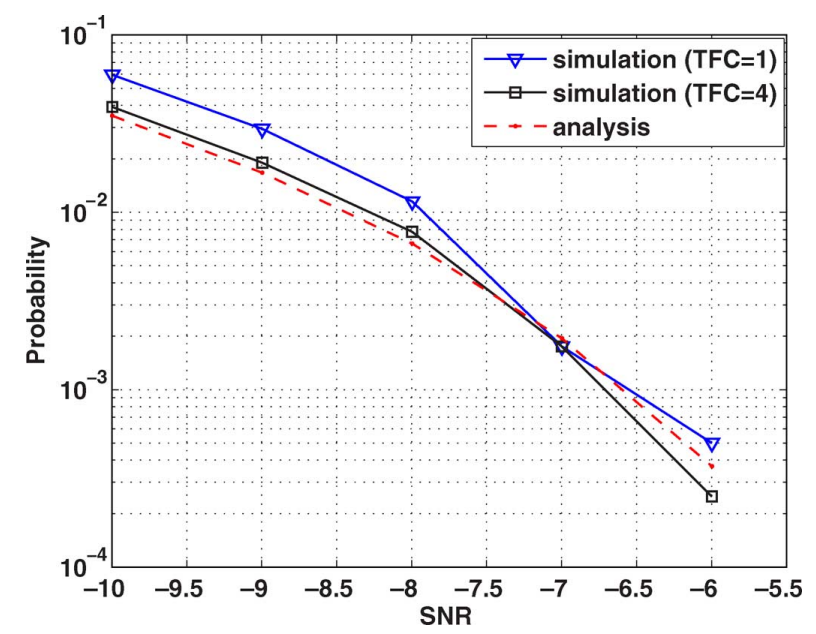

Fig. 7. The TFC identification error probabilities of using band-switching, given that the correct TFC group has been identified in signal detection stage, in UWB CM1 channels. The analytical result (19) is compared to the simulation results.

the probability of incorrectly identifying the TFC is equivalent to the probability of making the wrong decision on the position of the ACF output peak, which is given by

$$
\begin{aligned}
P\left[X_{1}<X_{3}\right] & =\int_{0}^{\infty} P\left[X_{3}>x \mid x\right] f_{X_{1}}(x) d x \\
& \approx \frac{1}{2\left(\kappa S N R_{r}+1\right)} \exp \left[-\frac{\left(N+N_{g}\right) \kappa^{2} S N R_{r}^{2}}{2\left(\kappa S N R_{r}+1\right)}\right],
\end{aligned}
$$

where $\kappa=3$. When $S N R_{r} \ll 1$, the decrease of the error probability is approximately proportional to $\exp \left[-c_{1} S N R_{r}^{2}\right]$ where the constant $c_{1} \triangleq 9\left(N+N_{g}\right) / 2$; when $S N R_{r} \gg 1$, the decrease of the error probability is approximately proportional to $S N R_{r}^{-1} \exp \left[-c_{2} S N R_{r}\right]$ where $c_{2} \triangleq c_{1} / 3$. The effectiveness of (19) is validated by simulation shown in Fig. 7.

We then compare the performance of the proposed ACF-based parallel signal detector with a conventional CCF-based one. Fig. 8 shows the miss detection probabilities for both schemes under different false alarm rate requirements $P_{f}^{t h}$, with UWB CM1 channels and zero CFO. The detection threshold (relative to the peak value of the corresponding detector output) for a given $P_{f}^{t h}$ for each scheme has been optimized via a numerical search over the range $(0,1]$. Except 


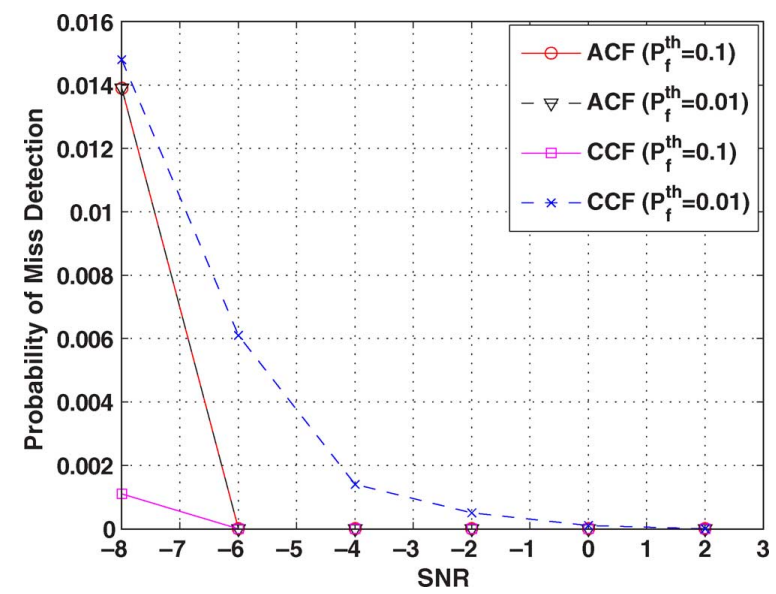

Fig. 8. Miss detection probabilities of the ACF-based parallel signal detector and a CCF-based signal detector under different false alarm rates $P_{f}^{t h}=\{0.1,0.01\}$, with TFC 1 and in UWB CM1 channels. The "zero" miss detection probability indicates that the actual probability is less than $5 \times 10^{-5}$.

the case of extremely low SNRs (e.g., $-8 \mathrm{~dB}$ ), the performance of the ACF-based parallel signal detector is same or better than the CCF-based one. Given that the practically interested SNR for the operation of the MB-OFDM UWB system is no less than $-6 \mathrm{~dB}$ [1], the ACF-based parallel signal detector is as effective as the CCF-based one. In addition, ACF-based detectors are much less sensitive to $\mathrm{CFO}$ effect than CCF-based ones [19].

The comparison on the computational complexity between signal detection schemes is presented in Table III, where, besides the conventional CCF-based scheme, a recently proposed ACF-based scheme in [23] has also been included in complexity comparison. As we are unable to find any existing work on TFC identification in the literatures, we assume that the TFC of the received signal is known in all schemes under the comparison. Thus, in the CCF-based scheme, the TFC specific $N$-sample time domain sequence of a synchronization symbol is used to cross-correlate with the received samples. In the proposed parallel ACF signal detection, since the TFC of the received signal is known, only two ACF units are active during the TFC identification stage. For example, if TFC is 1 or 2 , only the second and the fourth ACF units are needed to keep active (ref. Table I). Suppose that the signal detection is successful with a search interval of $W_{d} T$ in all schemes. By noting that one complex multiplication operation can be achieved with three real multiplication operations and five real additions in implementation [29], we obtain the computational complexity results in Table III, where $N^{\prime} \in\left[N, N+N_{g}\right]$ is the number of samples in a segment in an ACF operation, $\Delta(\geq 1)$ is a design parameter in the scheme in [23], MULT/ADD are real multiplication/addition, $\mathrm{ABS}$ stands for the operation of obtaining the amplitude of a complex number and COMP is the comparison of two real numbers. In practice, as the value of $W_{d}$ is usually large, e.g., close to or greater than $N(=128)$, the computation cost of the CCF-based detection scheme is much higher than the ACF-based schemes. Although the detection scheme in [23], which employs a single ACF unit, incurs the minimum computation cost among all schemes, it would fail in certain scenarios. For example, since the standard mandates a time-domain
TABLE III

The Comparison on Computational CompleXity of Signal DeteCtion SCHEME

\begin{tabular}{c||c|c|c}
\hline Operation & Proposed & CCF-based & Scheme in [23] \\
\hline \hline \# MULT & $6\left(N^{\prime}+W_{d}-1\right)$ & $2 W_{d} N$ & $3\left(N^{\prime}+W_{d}-1\right)$ \\
\# ADD & $2\left(7 N^{\prime}+4 W_{d}-6\right)$ & $2 W_{d}(N-1)$ & $7 N^{\prime}+4 W_{d}-6$ \\
\# ABS & $2 W_{d}$ & $W_{d}$ & $\left\lceil W_{d} / \Delta\right\rceil$ \\
\# COMP & $2 W_{d}$ & $W_{d}$ & $\left\lceil W_{d} / \Delta\right\rceil$ \\
\hline
\end{tabular}

spreading on all data symbols (i.e., each OFDM symbol is duplicated) when the data rate is $200 \mathrm{Mbps}$ or lower [1], the scheme in [23] is unable to distinguish preamble symbols and data symbols when TFC 3 or 4 is selected.

The good performance and the significant computation saving with ACF operations as discussed in the above, would make our ACF-based parallel signal detector more attractive than conventional detectors in practice.

\section{B. The Evaluation of CFO Estimation and Symbol Timing}

Fig. 9(a) and (b) show the simulated residual CFOs (i.e., $E[|\Delta \hat{f}-\Delta f|])$ of the proposed iterative CFO estimator after the first three iterations. The initial CFO is set as $40 \mathrm{ppm}$ at the frequency $3.960 \mathrm{GHz}$. Different UWB channels (i.e., CM1, CM4) and TFC settings (i.e., TFC 1 and TFC 4) are simulated. The values of $p$ in three iterations are given as $p=\{3,6,12\}$ in TFC 1 and $p=\{1,6,12\}$ in TFC 4 . The stepsize $\alpha=1$. Three other conventional schemes are also simulated for comparison. The first scheme is the $\mathrm{CFO}$ estimation based on the ACF values with a single value of $p$ (i.e., $p=3$ in TFC 1 and $p=1$ in TFC 4), which is equivalent to the iterative CFO estimation with one iteration. We denote this scheme as "Single ACF". The second scheme is the CFO estimation based on the ACF outputs with different values of $p$, in which the estimated CFO based on ACF of different $p$ are averaged to improve the CFO estimation. We denote this scheme as "Average ACF". The last scheme is proposed in [21] and is similar to the second scheme. It differs from the "Average ACF" in that the linear combination of individual estimates is based on the BLUE principle 7 . We can conclude from the results in Fig. 9(a) and (b) that first of all, the proposed iterative CFO estimator achieves the best performance among all schemes with the available ACF values; and secondly, two iterations in the iterative CFO estimation (i.e., $p=\{3,6\}$ in TFC 1 , or $p=\{1,6\}$ in TFC 4 ) is sufficient to reduce the residual CFO to $1 \sim 2 \mathrm{ppm}$ even when SNR is as low as $-3 \mathrm{~dB}$.

Appendix $\mathrm{C}$ gives the detailed analysis on the performance of the proposed iterative CFO estimation scheme. Using (23) and (30), and note that $S N R_{r} \approx\left(A_{d} / \kappa\left(N+N_{g}\right) \sigma_{\nu}^{2}\right)$, we can show that the expected value of residual $\mathrm{CFO}$ is

$$
E[|\Delta \hat{f}-\Delta f|] \approx \frac{\sqrt{\left(2+S N R_{r}^{-1} / \kappa\right) S N R_{r}^{-1} / \kappa}}{2 \pi^{3 / 2} p N_{s} \sqrt{N+N_{g}} T},
$$

${ }^{7}$ The proposed iterative CFO estimator is nonlinear. In step (2a)-(2b) in Table II, arg $\{\cdot\}$ operation performed over the estimated CFO in the previous iteration is an nonlinear operation. 

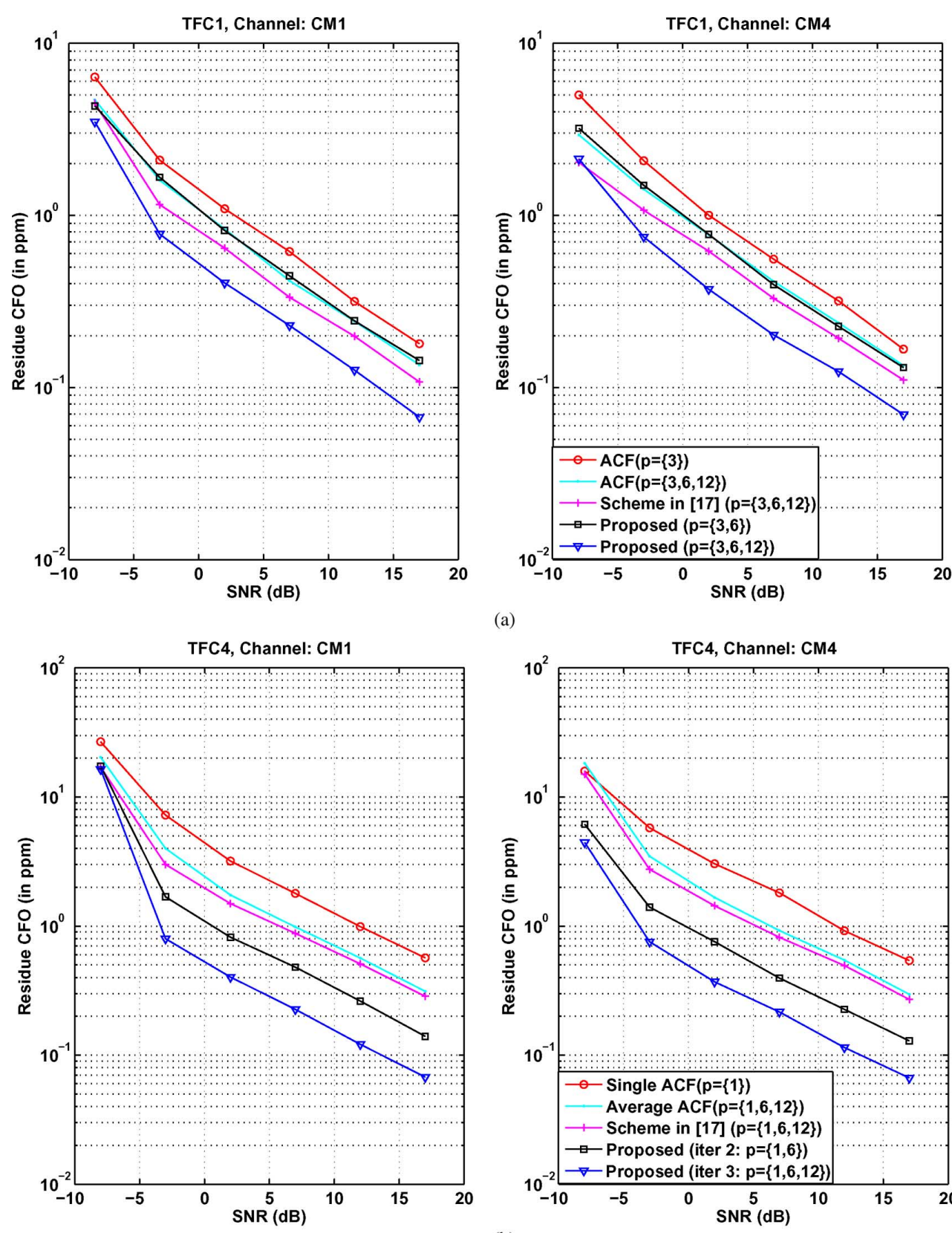

(a)

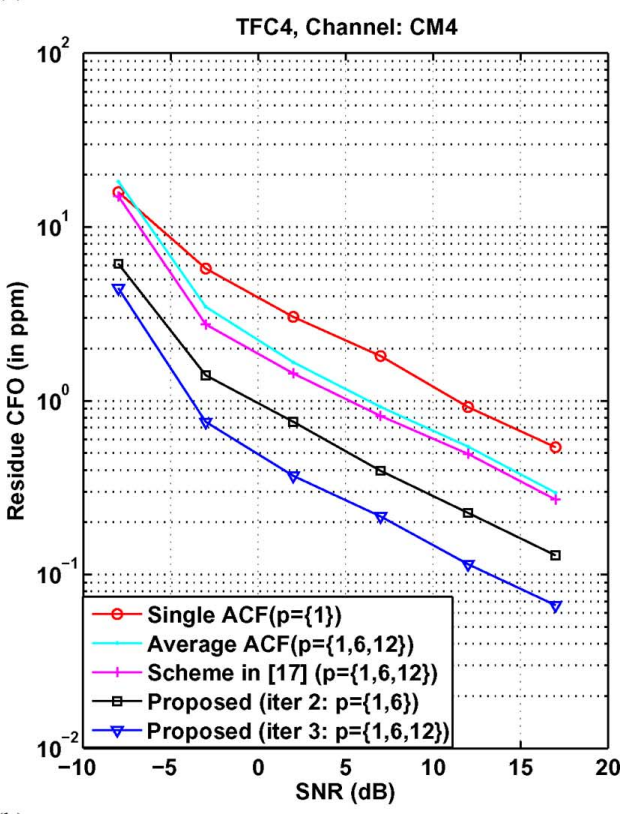

(b)

Fig. 9. The residual CFO $E[|\Delta \hat{f}-\Delta f|]$ (in ppm) during the first three iterations of the iterative CFO estimation, with TFC 1 and TFC 4 in UWB CM1 and CM4 channels, respectively. The conventional CFO estimation schemes are compared. The initial CFO is set as $40 \mathrm{ppm}$ at $3.960 \mathrm{GHz}$. The stepsize $\alpha=1$. Note that the Single ACF scheme with $p=3(p=1)$ in TFC 1 (TFC 4$)$ is the same as the proposed iterative CFO estimation in the first iteration.

which implies that $E[|\Delta \hat{f}-\Delta f|]$ decreases at the rate of $S N R_{r}^{-1 / 2}$ when $S N R_{r} \gg(1 / 2 \kappa)$ (e.g., $S N R_{r} \gg-7.8 \mathrm{~dB}$ for the case TFC 1-4 where $\kappa=3$ ). The simulation in Fig. 10 confirms the effectiveness of (20) where the slopes of residual $\mathrm{CFO}$ decrease in different iterations of the simulation match the prediction by analysis when $S N R_{r} \geq 0 \mathrm{~dB}$. Eqn. (20) also indicates that the decrease of residual $\mathrm{CFO}$ is proportional to $1 / p$. Thus the algorithm in Table II requires that $p_{k}>p_{k-1}$ to ensure a decrease of the expected residual CFO in each iteration. From Fig. 10, we also observe a significant performance improvement with iterations in $\mathrm{CFO}$ estimation. Compared to non-iterative CFO estimation (i.e., the estimation of "iter 1" in Fig. 10), one additional iteration (i.e., "iter 2") results in about
$10 \mathrm{~dB}$ improvement over the non-iterative estimation in TFC 4 , when $S N R_{r} \geq-3 \mathrm{~dB}$. An additional $4 \sim 5 \mathrm{~dB}$ gain is achieved with a third iteration.

Fig. 11 shows the uncoded BER performance of the MB-OFDM UWB receiver using the proposed CFO estimator and the timing metric (9) in both UWB CM1 and CM4 channels. The initial CFO is set as $40 \mathrm{ppm}$ at the frequency 3.960 GHz. For comparison, we also show the BER performance of an ideal receiver with zero residue CFO and the optimal timing in (6). The near-optimal performance of the proposed timing and CFO estimation demonstrates the effectiveness of our design and also validates the analysis on the timing metric (9) in Section IV-C. In addition, the timing and CFO 
TABLE IV

THE COMPARISON ON COMPUTATIONAL COMPLEXITY OF SyMBOL TIMING SCHEMES

\begin{tabular}{c||c|c|c|c|c}
\hline Operation & Proposed (MC [14]) & Scheme in [23] & MMSE [14] & ML [14] & Schmidl \& Cox [11] \\
\hline \hline \# MULT & $3 H_{t}\left(N^{\prime}+W_{t}-1\right)$ & $3 H_{t}\left(N^{\prime}+W_{t}-1\right)$ & $3 H_{t}\left(N^{\prime}+W_{t}-1\right)$ & $3 H_{t}\left(N^{\prime}+W_{t}-1\right)$ & $3 H_{t}\left(N^{\prime}+W_{t}-1\right)$ \\
& & & $+4 H\left(N^{\prime}+W_{t}-1\right)$ & $+4 H\left(N^{\prime}+W_{t}-1\right)$ & $+2 H\left(N^{\prime}+W_{t}-1\right)$ \\
& & & & $+W_{t}$ & \\
\# ADD & $H_{t}\left(7 N^{\prime}+4 W_{d}-6\right)$ & $H_{t}\left(7 N^{\prime}+4 W_{d}-6\right)$ & $H_{t}\left(7 N^{\prime}+4 W_{d}-6\right)$ & $H_{t}\left(7 N^{\prime}+4 W_{d}-6\right)$ & $H_{t}\left(7 N^{\prime}+4 W_{d}-6\right)$ \\
& $+W_{t}(H-1)$ & $+W_{t}\left(H_{t}-1\right)$ & $+W_{t}(4 H-1)$ & $+W_{t}(4 H-1)$ & $+W_{t}(H-1)$ \\
& & $+\left\lceil W_{d} / \Delta_{1}\right\rceil\left(H_{d}-1\right)$ & $+2 H\left(2 N^{\prime}+3 W_{t}-4\right)$ & $+2 H\left(2 N^{\prime}+3 W_{t}-4\right)$ & $+H\left(2 N^{\prime}+3 W_{t}-4\right)$ \\
\# ABS & $W_{t} H_{t}$ & $W_{t} H_{t}$ & $W_{t} H_{t}$ & $W_{t} H_{t}$ & $W_{t} H_{t}$ \\
\# COMP & $W_{t}-1$ & $\left\lceil W_{d} / \Delta_{1}\right\rceil+W_{t}-2$ & $W_{t}-1$ & $W_{t}-1$ & $W_{t}-1$ \\
\# DIV & - & - & - & - & $W_{t} H$ \\
\hline
\end{tabular}

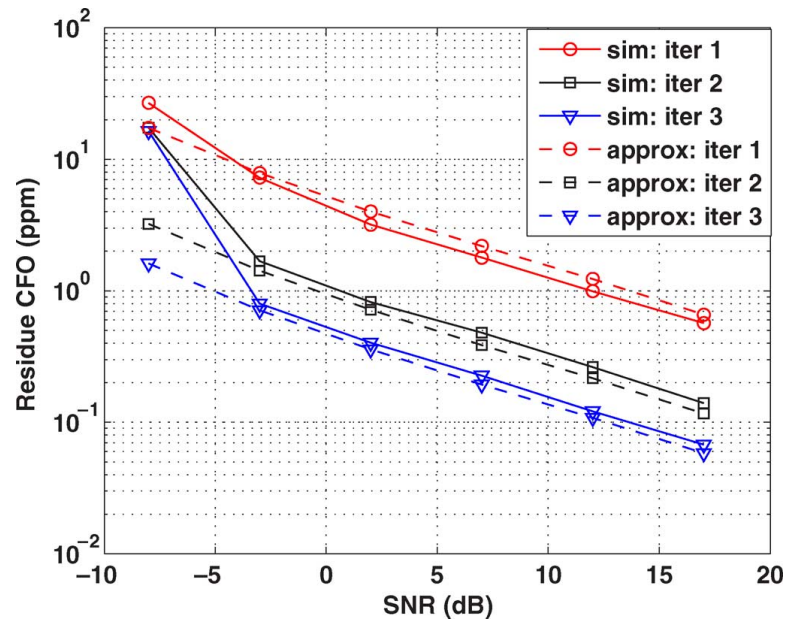

Fig. 10. Residual $\mathrm{CFO}$ (in ppm) in the first three iterations in the iterative $\mathrm{CFO}$ estimation and correction, compared to the approximation in (20), with TFC 4 and in UWB CM1 channels. The stepsize $\alpha=1$.

estimation for the BER performance in Fig. 11 only uses the ACF values which are already available in signal detection and TFC identification stage and therefore it minimizes the extra resources needed in computation.

To further evaluate the complexity of the symbol timing and iterative CFO estimation in the proposed SYNC design, we compare our design with the conventional ones in the literature.

1) For symbol timing, we consider different ACF-based metrics used in [11], [14], [23]. Let $H=\left(H_{d}+H_{t}\right)$ be the ACF outputs that are used for symbol timing, where $H_{d}(\geq 1)$ ACF outputs are already available from signal detection and TFC identification stage and an additional $H_{t}(\geq 0)$ ACF outputs are calculated in the symbol timing stage. We further assume that the timing point search window size is $W_{t}$ samples. Table IV lists the computational complexity for each timing scheme, where $N^{\prime} \in[N, N+$ $\left.N_{g}\right]$ is the length of signal segments in an ACF operation, $\Delta_{1}(\geq 1)$ is a design parameter in the scheme in [23], DIV stands for real division. Table IV shows that the computation costs of MC metric used in the proposed SYNC design and the metric used in [23] are similar and both are much lower than the metrics with minimum mean-squared-error (MMSE) criterion, maximum likelihood (ML) criterion as well as the metric proposed in [11].

2) For CFO estimation, we compare the computational complexity of all the schemes used in the performance comparison [shown in Fig. 9(a)]. Note that there are

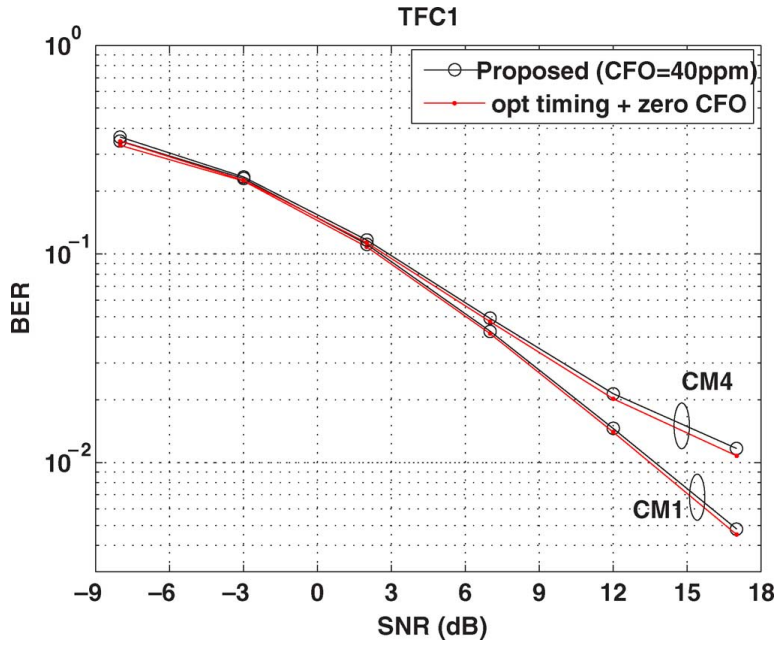

Fig. 11. Demodulated BER performance of the receiver with the proposed CFO estimation (2 iterations) and the symbol timing metric (9), compared to the one with zero CFO and the optimal timing point which maximizes the SNR at the output of the FFT operation, in UWB CM1 and CM4 channels, respectively. The initial CFO is set to be $40 \mathrm{ppm}$ at $3.960 \mathrm{GHz}$.

$H$ ACF outputs available at the symbol timing stage. Suppose that there are $H_{c}(\leq H)$ of these ACF outputs with different values of $p$ and we use these ACF outputs for CFO estimation purpose. Due to the possible phase ambiguity in CFO estimation with the scheme in [21] as well as the "Average ACF" scheme, a phase compensation based on the initial CFO estimation with the smallest value of $p$ should be carried out as suggested in [21]. For the scheme in [21], the computation of the weights of individual estimates is assumed to be carried out off-line with a nominal SNR and its cost is not included in our estimation. Table V shows the computational complexity of each scheme, where ARG is the operation to extract the angular component of a complex number. Two versions of the scheme proposed in [21] are evaluated in the table. Although the "Single ACF" scheme achieves the lowest computation cost among all schemes, it also has the worst performance [see Fig. 9(a) and (b)]. The proposed iterative CFO estimation incurs the second lowest computation cost with the best performance among all schemes.

\section{The Evaluation of the NBI Frequency Detector}

To evaluate the performance of the proposed frequency detector, we consider a modulated NBI signal with a data rate 1.6 
TABLE V

THE COMPARISON ON COMPUTATIONAL COMPLEXITY OF CFO ESTIMATION SCHEMES

\begin{tabular}{c||c|c|c|c|c}
\hline Operation & Proposed & $\begin{array}{c}\text { Scheme in [21] } \\
\text { w/ energy weighting }\end{array}$ & $\begin{array}{c}\text { Scheme in [21] } \\
\text { w/o energy weighting }\end{array}$ & Single ACF & Average ACF \\
\hline \hline \# MULT & $5 H_{c}-4$ & $6\left(N^{\prime}+H_{c}\right)+11$ & $6 H_{c}+5$ & 0 & $5 H_{c}-3$ \\
\# ADD & $6 H_{c}-6$ & $6\left(N^{\prime}+H_{c}\right)-7$ & $6 H_{c}-4$ & 1 & $6 H_{c}-6$ \\
\# ARG & $H_{c}$ & $H_{c}$ & $H_{c}$ & 1 & $H_{c}$ \\
\# DIV & - & 1 & 1 & - & - \\
\hline
\end{tabular}

TABLE VI

THE ABSOLUTE ERRor (With 95\% CONFIDENCE LEVEL) OF NBI FREQUENCY ESTIMATION, NORMALIZED BY THE INTER-SUBCARRIER SPACING IN MB-OFDM UWB (SIR $=0 \mathrm{~dB}$, TFC- $1, \beta=1)$

\begin{tabular}{c||c|c}
\hline INR $(\mathrm{dB})$ & in CM1 Channels & in CM4 Channels \\
\hline \hline-9 & $13.4311 \pm 1.6290$ & $15.8030 \pm 1.8229$ \\
-4 & $7.5120 \pm 1.3621$ & $7.4037 \pm 1.3976$ \\
1 & $2.7367 \pm 0.9576$ & $2.7944 \pm 0.9604$ \\
6 & $0.1887 \pm 0.2503$ & $0.5744 \pm 0.4905$ \\
11 & $0.1566 \pm 0.2515$ & $0.1579 \pm 0.2512$ \\
16 & $0.0186 \pm 0.0015$ & $0.1470 \pm 0.2513$ \\
\hline
\end{tabular}

Mbps in the first sub-band (i.e., 3.168-3.696 GHz). Its central frequency is randomly selected in the sub-band and $S I R$ is set as $0 \mathrm{~dB}$. The TFC of the MB-OFDM UWB signal is set as 1 . The stepsize $\beta=1$. Table VI summarizes the simulation results. In the table, the (absolute) estimation error on frequency is normalized to the inter-subcarrier spacing (i.e., 4.125 MHz). We observe that when interference-to-noise-ratio (INR) is above $5 \mathrm{~dB}$, i.e., the NBI is dominant, the estimated NB central frequency is within one tone of the actual NBI frequency with high probability (i.e., 95\%). The designed notch filter can thus eliminate the effect of NBI by removing or suppressing the received signal within $2 \sim 4$ subcarriers of the detected frequency [15]. The loss of information on these subcarriers has little effect on synchronization stage. Its impact on data demodulation is mitigated by the interleaving and error-correct coding schemes specified in the standard [1]. It is also possible that the NBI frequency information be fed back to the transmitter to enable the detection and avoidance that are enforced in some countries for all UWB systems.

\section{CONCLUSIONS}

In this paper, we proposed a synchronization architecture for MB-OFDM UWB systems featuring a parallel ACF structure and functional blocks designed sharing the parallel ACF outputs. This design fully exploited the correlation of the designs of individual functional blocks in the synchronizer and thus reduces complexity by sharing a large portion of computation burden among different functional blocks. The key features of our design include: (i) a joint signal detection and TFC identification with the proposed parallel ACF structure that has a low computation cost, fast acquisition and non-compromised performance; (ii) a symbol timing with the maximum correlation (MC) metric that can achieve a near-optimal performance, confirmed by our analysis and simulation; (iii) an iterative structure for CFO estimation that improves both the range and the accuracy of the estimation. We have also presented a low-complexity NBI frequency detector which can enhance the performance of the synchronizer in a strong NBI scenario. Most importantly, the proposed synchronization architecture and individual functional block design can be combined with the existing various hardware-oriented implementation techniques to achieve a desired performance and complexity in the actual system.

\section{APPENDIX A \\ THE DERIVATION FOR EQN. (10)}

Consider the timing metric in (9) and the approximations

$$
\begin{aligned}
& \left|A C\left(d p, N_{g}\right)\right| \\
& \left.\approx\left|\sum_{i}\right| h_{i}\right|^{2} \sum_{m=0}^{N+N_{g}-1}|s[m+d-i]|^{2} \\
& \quad+\sum_{i} \sum_{j \neq i} h_{i}^{*} h_{]} j \sum_{m=0}^{N+N_{g}-1} s^{*}[m+d-i] s[m+d-j] \mid \\
& \approx \sum_{i}\left|h_{i}\right|^{2} \sum_{m=0}^{N+N_{g}-1}|s[m+d-i]|^{2},
\end{aligned}
$$

where timing bias $d$ is around $\arg \max _{d}\left\{\left|A C\left(d ; p, N_{g}\right)\right|\right\}$ and the multipath delay $\tau_{i}=i T$. The first approximation comes from that uncorrelated noise samples results negligible composite noise terms when $d$ is near the peak of the ACF metric; and the second approximation is due to the pseudo-random (PR) property of the 128-sample sequence of a synchronization symbol, which makes $\sum_{m=0}^{N+N_{g}-1} s^{*}[m+d-i] s[m+d-j]$, $i \neq j$ negligible when $d$ is close to the peak of the ACF metric.

Let $P_{N+N_{g}}(d) \triangleq \sum_{m=0}^{N+N_{g}-1}|s[m+d]|^{2}$, we note that shape of $P_{N+N_{g}}(d)$ for any TFC specified PR sequence $\{s[m]\}$ in [1] is close to

$$
\tilde{P}_{N+N_{g}}(\eta)= \begin{cases}-\eta+N T & 0 \leq \eta \leq N T \\ N T & -T_{g} \leq \eta<0 \\ \eta+\left(N+N_{g}\right) T & -\left(N+N_{g}\right) T \leq \eta<T_{g}\end{cases}
$$

where $\eta$ is the timing bias in continuous time domain. Thus, the timing metric in (9) approximately maximizes

$$
\int_{\eta-N T}^{\eta+\left(N+N_{g}\right) T}|h(\tau)|^{2} \tilde{P}_{N+N_{g}}(\eta-\tau) d \tau
$$

with $d$ being the closest integer to $\eta / T$. To find the optimal value of $\eta^{*}$ that maximizes (21), it is sufficient to only consider the range $\eta \in\left(-\left(N_{g}+N\right) T+\tau_{\max }, N T\right)$. It can be shown that the maximization in (21) is equivalent to minimize the sum of $\int_{\eta+T_{g}}^{\eta+\left(N+N_{g}\right) T}|h(\tau)|^{2}\left(\tau-\eta-T_{g}\right) d \tau$ and $\int_{\eta-N T}^{\eta}|h(\tau)|^{2}(\eta-$ $\tau) d \tau$ over $\eta$. By taking $\partial / \partial \eta=0$ and observing that $h(\eta-$ 
$N T)=h\left(\eta+\left(N+N_{g}\right) T\right)=0$, for $\eta \in\left(-\left(N_{g}+N\right) T+\right.$ $\left.\tau_{\text {max }}, N T\right)$, we find that the optimal $\eta^{*}$ satisfies

$$
\int_{\eta^{*}-N T}^{\eta^{*}}|h(\tau)|^{2} d \tau=\int_{\eta^{*}+T_{g}}^{\eta^{*}+\left(N+N_{g}\right) T}|h(\tau)|^{2} d \tau .
$$

Then (10) follows by observing that $h(\tau)=0$, for $\tau<0$ and $\tau>\tau_{\text {max }}$ in the above equality, and $\hat{d}_{1}$ as the closest integer to $\eta^{*} / T$.

\section{APPENDIX B}

The Distributions of $\left|A C\left(d ; p, N_{g}\right)\right|$

In the ACF-based parallel signal detector, two received signal segments in an ACF operation are separated by $p N_{s} T$, where $p=1,3,5,6$. There are three cases for the inputs in an ACF operation:

1) the synchronization symbols present in both segments of the ACF operation;

2) the synchronization symbol only presents in one of two signal segments of the ACF operation;

3) no synchronization symbol presents in either signal segments of the ACF operation.

For different cases, the output of the ACF operation has different distributions and the detection capability of the proposed parallel signal detector is determined by these distributions. We consider the signal segments with the length $N+N_{g}=160$ in an ACF operation to analyze the performance of the signal detector. The ACF operation on the signal segments with other lengths can also be analyzed by the same procedure presented here. To be concise, we neglect the possible phase rotation in $A C\left(d ; p, N_{g}\right)$, caused by the CFO between the transmitter and the receiver, since the detection is solely determined by the amplitude of $A C\left(d ; p, N_{g}\right)$.

In case 1 , when the synchronization symbols present in both signal segments of the ACF operation,

$$
A C\left(d p, N_{g}\right)=\sum_{m=0}^{N+N_{g}-1}\left|\sum_{i} h_{i} s[m+d-i]\right|^{2}+\nu_{d},
$$

where the composite noise $\nu_{d}$ is given by

$$
\begin{aligned}
\nu_{d}= & \sum_{i} h_{i}^{*} \sum_{m} s^{*}[m+d-i] \nu\left[m+p N_{s}+d\right] \\
& +\sum_{i} h_{i} \sum_{m} s[m+d-i] \nu^{*}[m+d] \\
& +\sum_{m} \nu^{*}[m+d] \nu\left[m+p N_{s}+d\right] .
\end{aligned}
$$

Let

$$
A_{d} \triangleq \sum_{m}\left|\sum_{i} h_{i} s[m+d-i]\right|^{2},
$$

for a given channel realization $\left\{h_{i}\right\}$, it is straightforward to show that the variance of the composite noise $\nu_{d}$ is

$$
\sigma_{\nu_{d}}^{2}=\left[2 A_{d}+\left(N+N_{g}\right) \sigma_{\nu}^{2}\right] \sigma_{\nu}^{2}
$$

Furthermore, since the range of summation $\sum_{m}$ is large, i.e., $N+N_{g}=160$, according to the Central-Limit-Theorem, the composite noise is approximately a zero-mean complex Gaussian random variable, i.e., $\nu_{d} \sim \mathcal{C N}\left(0, \sigma_{\nu_{d}}^{2}\right)$. Correspondingly, $A C\left(d ; p, N_{g}\right) \sim \mathcal{C N}\left(A_{d}, \sigma_{\nu_{d}}^{2}\right)$. Let $A C\left(d ; p, N_{g}\right)=$ $\mathcal{R}_{p, d}+j \mathcal{I}_{p, d}$, it can be shown that $\mathcal{R}_{p, d} \sim \mathcal{N}\left(A_{d}, \sigma_{\nu_{d}}^{2} / 2\right)$ and $\mathcal{I}_{p, d} \sim \mathcal{N}\left(0, \sigma_{\nu_{d}}^{2} / 2\right)$. From the definitions of $\mathcal{R}_{p, d}$ and $\mathcal{I}_{p, d}$, we have $E\left[\mathcal{R}_{p, d} \mathcal{I}_{p, d}\right]=E\left[\mathcal{R}_{p, d}\right] E\left[\mathcal{I}_{p, d}\right]=0$, i.e., $\mathcal{R}_{p, d}$ and $\mathcal{I}_{p, d}$ are uncorrelated. Since $\mathcal{R}_{p, d}$ and $\mathcal{I}_{p, d}$ are (approximately) Gaussian, $\mathcal{R}_{p, d}$ and $\mathcal{I}_{p, d}$ are (approximately) independent.

The detection on an ACF value is based on comparing $X_{1} \triangleq\left|A C\left(d ; p, N_{g}\right)\right|$ with a given threshold $\lambda(>0) . X_{1}$ follows a Rice distribution, i.e., the probability distribution function (PDF) of $X_{1}$ is given by

$$
f_{X_{1}}(x)=\frac{x}{\sigma_{\nu_{d}}^{2} / 2} \exp \left[-\frac{x^{2}+A_{d}^{2}}{\sigma_{\nu_{d}}^{2}}\right] I_{0}\left(\frac{A_{d} x}{\sigma_{\nu_{d}}^{2} / 2}\right),
$$

where $I_{0}(\cdot)$ is the zero-order modified Bessel function of the first kind.

In case 2 , when the synchronization symbol only presents in one of two signal segments of the ACF operation,

$$
\begin{aligned}
A C\left(d ; p, N_{g}\right)= & \sum_{m=0}^{N+N_{g}-1}\left\{\sum_{i} h_{i}^{*} s^{*}[m+d-i]+n^{*}[m+d]\right\} \\
& \times n\left[m+p N_{s}+d\right],
\end{aligned}
$$

where we assume that the synchronization symbol presents at the first signal segment of the ACF operation but there is no difference by assuming the synchronization symbol presenting in the second signal segment because of the commutativity of the operation. It can be shown that $A C\left(d ; p, N_{g}\right)=\mathcal{R}_{p, d}+j \mathcal{I}_{p, d}$ where $\mathcal{R}_{p, d} \sim \mathcal{N}\left(0,\left[A_{d}+\left(N+N_{g}\right) \sigma_{\nu}^{2}\right] \sigma_{\nu}^{2} / 2\right)$ and $\mathcal{I}_{p, d} \sim \mathcal{N}\left(0,\left[A_{d}+\left(N+N_{g}\right) \sigma_{\nu}^{2}\right] \sigma_{\nu}^{2} / 2\right)$. From the definitions of $\mathcal{R}_{p, d}$ and $\mathcal{I}_{p, d}$, we can show that they are (approximately) independent. Correspondingly, $X_{2} \triangleq\left|A C\left(d ; p, N_{g}\right)\right|$ follows a Rayleigh distribution, i.e., the PDF of $X_{2}$ is given by

$$
\begin{aligned}
f_{X_{2}}(x)= & \frac{x}{\left[A_{d}+\left(N+N_{g}\right) \sigma_{\nu}^{2}\right] \sigma_{\nu}^{2} / 2} \\
& \times \exp \left\{\frac{-x^{2}}{\left[A_{d}+\left(N+N_{g}\right) \sigma_{\nu}^{2}\right] \sigma_{\nu}^{2}}\right\} .
\end{aligned}
$$

In case 3, when there is no synchronization symbol in either signal segment of the ACF operation,

$$
A C\left(d p, N_{g}\right)=\sum_{m=0}^{N+N_{g}-1} n^{*}[m+d] n\left[m+p N_{s}+d\right] .
$$

It can be shown that $A C\left(d ; p, N_{g}\right)=\mathcal{R}_{p, d}+j \mathcal{I}_{p, d}$ where $\mathcal{R}_{p, d} \sim \mathcal{N}\left(0,\left(N+N_{g}\right) \sigma_{\nu}^{4} / 2\right)$ and $\mathcal{I}_{p, d} \sim$ $\mathcal{N}\left(0,\left(N+N_{g}\right) \sigma_{\nu}^{4} / 2\right)$. $\mathcal{R}_{p, d}$ and $\mathcal{I}_{p, d}$ are approximately independent. Correspondingly, $X_{3} \triangleq\left|A C\left(d ; p, N_{g}\right)\right|$ follows a Rayleigh distribution, i.e., the PDF of $X_{3}$ is

$$
f_{X_{3}}(x)=\frac{x}{\left(N+N_{g}\right) \sigma_{\nu}^{4} / 2} \exp \left\{-\frac{x^{2}}{\left(N+N_{g}\right) \sigma_{\nu}^{4}}\right\} .
$$


Assume that the true frequency offset $\Delta f$ is deterministic but unknown. The estimated CFO in the $(k-1)^{t h}$ iteration $(k \geq 2)$ is $\Delta \hat{f}_{k-1}$ and let the step size $\alpha=1$. The interval between two signal segments in current ACF operation is $p_{k} N_{s} T$ and the ACF output value is

$$
\begin{aligned}
& A C\left(d p_{k}, N_{g}\right) \\
& \quad=e^{j 2 \pi p_{k} N_{s} \Delta f T} \sum_{m=0}^{N+N_{g}-1}\left|\sum_{i} h_{i} s[m+d-i]\right|^{2}+\nu_{d}^{\prime} \\
& \quad=A_{d} e^{j 2 \pi p_{k} N_{s} \Delta f T}+\nu_{d}^{\prime},
\end{aligned}
$$

where $A_{d}$ is given in (22), $d$ is around $\arg \max _{d}\left\{\left|A C\left(d ; p_{k}, N_{g}\right)\right|\right\} \quad$ and the composite noise

$$
\begin{aligned}
\nu_{d}^{\prime}= & \sum_{i} h_{i}^{*} \sum_{m} e^{-j 2 \pi \Delta f(m+d) T} s^{*}[m+d-i] \nu\left[m+p_{k} N_{s}+d\right] \\
& +\sum_{i} h_{i} \sum_{m} e^{j 2 \pi \Delta f\left(m+p_{k} N_{s}+d\right) T s}[m+d-i] \nu^{*}[m+d] \\
& +\sum_{m} \nu^{*}[m+d] \nu\left[m+p_{k} N_{s}+d\right] .
\end{aligned}
$$

Similar to the argument for $\nu_{d}$ in Appendix B, the composite noise $\nu_{d}^{\prime}$ is also approximately a zero-mean complex Gaussian random variable with variance given in (23).

In the $k^{\text {th }}$ iteration, the (absolute) residue CFO to be estimated is $\left|\Delta \hat{f}_{k}-\Delta f\right|$. Assuming only one ACF value is used in estimating the residue CFO, the output from the step (2b) in the algorithm (see Table II) is

$$
\delta \hat{f}_{k}=\frac{1}{2 \pi p_{k} N_{s} T} \arg \left\{A C\left(d p_{k}, N_{g}\right) e^{-j 2 \pi p_{k} N_{s} \Delta \hat{f}_{k-1} T}\right\}
$$

Note that when the timing point $d$ is close to the peak of ACF values, $S N R_{r} \approx\left(A_{d} / \kappa\left(N+N_{g}\right) \sigma_{\nu}^{2}\right)$. And with (23), it is readily to show that $\sigma_{\nu_{d}^{\prime}}=\sigma_{\nu_{d}} \approx$ $\left(\sqrt{2 \kappa S N R_{r}+1} / \kappa S N R_{r} \sqrt{N+N_{g}}\right) A_{d}$. Consider the case that $A_{d} \gg\left|\nu_{d}^{\prime}\right|$ with high probability. This is not an uncommon case in practice. For example, if $S N R_{r} \geq 0.41 \mathrm{~dB}$ in TFC 1-4 where $\kappa=3$, we can ensure that $A_{d} \geq 15.17 \sigma_{\nu_{d}}$ which indicates that $A_{d} \geq 10\left|\nu_{d}^{\prime}\right|$ with a probability ${ }^{8} 90 \%$. Geometrically, we can treat the noise vector $\left|\nu_{d}^{\prime}\right| e^{j\left(\theta_{\nu_{d}^{\prime}}-2 \pi p_{k} N_{s} \Delta \hat{f}_{k-1} T\right)}$ as a small disturbance around the point $A_{d} e^{j 2 \pi p_{k} N_{s}\left(\Delta f-\Delta \hat{f}_{k-1}\right) T}$ on the complex plane. Thus the following relationship holds

$$
\begin{aligned}
\arg & \left\{A C\left(d p_{k}, N_{g}\right) e^{-2 j \pi p_{k} N_{s} \Delta \hat{f}_{k-1} T}\right\} \\
\approx & 2 \pi p_{k} N_{s}\left(\Delta f-\Delta \hat{f}_{k-1}\right) T \\
& +\frac{\left|\nu_{d}^{\prime}\right| \sin \left[\theta_{\nu_{d}^{\prime}}-2 \pi p_{k} N_{s} \Delta f T\right]}{A_{d}+\left|\nu_{d}^{\prime}\right| \cos \left[\theta_{\nu_{d}^{\prime}}-2 \pi p_{k} N_{s} \Delta f T\right]}
\end{aligned}
$$

The estimation error after the $k^{t h}$ iteration is given by

$$
\begin{aligned}
& \left(\Delta \hat{f}_{k-1}+\delta \hat{f}_{k}\right)-\Delta f \\
& \quad \approx \frac{1}{2 \pi p_{k} N_{s} T} \frac{\left|\nu_{d}^{\prime}\right| \sin \left[\theta_{\nu_{d}^{\prime}}-2 \pi p_{k} N_{s} \Delta f T\right]}{A_{d}+\left|\nu_{d}^{\prime}\right| \cos \left[\theta_{\nu_{d}^{\prime}}-2 \pi p_{k} N_{s} \Delta f T\right]}
\end{aligned}
$$

${ }^{8}$ Note that $\left|\nu_{d}^{\prime}\right|$ approximately follows a Rayleigh distribution with the mode $\sigma_{\nu_{d}} / \sqrt{2}$.
Note that $\Delta \hat{f}_{k}=\Delta \hat{f}_{k-1}+\delta \hat{f}_{k}$, we can approximate the expected (absolute) residue $\mathrm{CFO}$ as

$$
E\left[\left|\Delta \hat{f}_{k}-\Delta f\right|\right] \approx \frac{E\left\{|| \nu_{d}^{\prime}\left|\sin \left[\theta_{\nu_{d}^{\prime}}-2 \pi p_{k} N_{s} \Delta f T\right]\right|\right\}}{2 \pi p_{k} N_{s} T A_{d}}
$$

where $\left|\nu_{d}^{\prime}\right| \sin \left[\theta_{\nu_{d}^{\prime}}-2 \pi p_{k} N_{s} \Delta f T\right]$ is (approximately) a (real) zero-mean Gaussian random variable with variance $\sigma_{\nu_{d}}^{2} / 2$. From (29), it is straightforward to see that

$$
E\left[\left|\Delta \hat{f}_{k}-\Delta f\right|\right] \approx \frac{\sigma_{\nu_{d}}}{2 \pi^{3 / 2} p_{k} N_{s} T A_{d}}
$$

\section{REFERENCES}

[1] High Rate Ultra-Wideband PHY and MAC Standard, Standard ECMA368, Dec. 2005, ECMA, 1st Ed..

[2] C. Duan, G. Pekhteryev, J. Fang, Y.-P. Nakache, J. Zhang, K. Tajima, Y. Nishioka, and H. Hirai, "Transmitting multiple HD video streams over UWB links," in Proc. IEEE Consumer Communications \& Networking Conf. (CCNC'06), Las Vigas, USA, Jan. 2006.

[3] X. Wang, T. T. Tjhung, Y. Wu, and B. Caron, "SER performance evaluation and optimization of OFDM system with residue frequency and timing offsets from imperfect synchronization," IEEE Trans. Broadcast., vol. 49, no. 2, pp. 170-177, Jun. 2003.

[4] M. Speth, S. A. Fechtel, G. Fock, and H. Meyr, "Optimum receiver design for wireless broad-band systems using OFDM-Part I," IEEE Trans. Commun., vol. 47, no. 11, pp. 1668-1677, Nov. 1999.

[5] M. Speth, S. A. Fechtel, G. Fock, and H. Meyr, "Optimum receiver design for OFDM-based broadband transmission-Part II: A case study," IEEE Trans. Commun., vol. 49, no. 4, pp. 571-578, Apr. 2001.

[6] G. Pantos, "A numerical technique for blind estimation of carrier frequency offset in OFDM systems," IEEE Trans. Broadcast., vol. 52, no. 4, pp. 566-569, Dec. 2006.

[7] A. J. Coulson, "Narrowband interference in pilot symbol assisted OFDM systems," IEEE Trans. Wireless Commun., vol. 3, no. 6, pp. 2277-2287, Nov. 2004.

[8] Z. Zhang, K. Long, M. Zhao, and Y. Liu, "Joint frame synchronization and frequency offset estimation in OFDM systems," IEEE Trans. Broadcast., vol. 51, no. 3, pp. 389-394, Sep. 2005.

[9] A. F. Molisch, J. R. Foerster, and M. Pendergrass, "Channel models for ultrawideband personal area networks," IEEE Personal Commun. Mag., no. 55, pp. 14-21, 2003.

[10] M. Speth, F. Classen, and H. Meyr, "Frame synchronization of OFDM systems in frequency selective fading channels," in Proc. IEEE Vehicular Technology Conf. (VTC'97), Phoenix, USA, 1997.

[11] T. Schmidl and D. Cox, "Robust frequency and timing synchronization for OFDM," IEEE Trans. Commun., vol. 45, no. 12, pp. 1613-1621, Dec. 1997.

[12] H. Minn, V. Bhargava, and K. Lataief, "A robust timing and frequency synchronization for OFDM systems," IEEE Trans. Wireless Commun., vol. 2, no. 4, pp. 822-838, Jul. 2003.

[13] J. G. Proakis, Digital Communications, 3rd ed. New York: McGrawHill, Inc., 1995.

[14] S. H. Muller-Weinfurtner, "On the optimality of metrics for coarse frame synchronization in OFDM: A comparison," in Proc. IEEE Int'l Symp. Personal, Indoor and Mobile Radio Communications (PIMRC'98), Boston, USA, Sep. 1998.

[15] K. Shi, Y. Zhou, B. Kelleci, T. W. Fischer, E. Serpedin, and A. Iker Karsilayan, "Impacts of narrowband interference on OFDM-UWB receivers: Analysis and mitigation," IEEE Trans. Signal Processing, vol. 55, no. 3, pp. 1118-1128, Mar. 2007.

[16] C. R. N. Athaudage and R. R. V. Angiras, "Sensitivity of FFT-equalised zero-padded OFDM systems to time and frequency synchronisation errors," IEE Proc. Commun., vol. 152, no. 6, pp. 945-951, Dec. 2005.

[17] H. Liu and C. Lee, "A low-complexity synchronizer for OFDM-based UWB system," IEEE Trans. Circuits \& Syst.-Part II, vol. 53, no. 11, pp. 1269-1273, Nov. 2006.

[18] H. Lee et al., "Efficient structures of packet/frame synchronization in MB-OFDM UWB," in Proc. IEEE Int'l Symp. Intelligent Signal Processing and Communication Systems (ISPACS '04), Seoul, Korea, Nov. 2004. 
[19] M. Krstic, A. Troya, K. Maharatna, and E. Grass, "Optimized lowpower synchronizer design for the IEEE 802.11(a) standard," in Proc. IEEE Int'l Conf. Acoustics, Speech, and Signal Processing (ICASSP '03), Hong Kong, China, Apr. 2003.

[20] F. Tufvesson, O. Edfors, and M. Faulkner, "Time and frequency synchronization for OFDM using PN-sequence preambles," in Proc. IEEE Vehicular Technology Conf. (VTC '99), Amsterdam, The Netherlands, Sep. 1999.

[21] Y. Li, T. Jacobs, and H. Minn, "Frequency offset estimation for MB-OFDM-based UWB systems," in Proc. IEEE Int'l Communications Conf. (ICC '06), Istanbul, Turkey, Jun. 2006.

[22] S. Yoon and J. Chong, "Packet detection and symbol timing synchronization algorithm for multi-band OFDM UWB," IEICE Trans. Commun., vol. E89-B, no. 4, pp. 1433-1435, Apr. 2006.

[23] T. Jacobs, Y. Li, and H. Minn, "Synchronization in MB-OFDM-based UWB systems," in Proc. IEEE Int'l Communications Conf. (ICC '07), Glasgow, Scotland, Jun. 2007.

[24] C. L. Wang and H. C. Wang, "An optimized joint time synchronization and channel estimation scheme for OFDM systems," in Proc. IEEE Vehicular Technology Conf. (VTC '08 Spring), Marina Bay, Singapore, May 2008

[25] D. Sen, S. Chakrabart, and R. V. Raja Kumar, "An efficient frequency offset estimation scheme for Multi-band OFDM ultra-wideband systems," in Proc. IEEE Vehicular Technology Conf. (VTC '08 Spring), Marina Bay, Singapore, May 2008.

[26] Y. Yao, X. Dong, and N. Tin, "A new joint timing and channel estimation method for block transmission UWB systems," in Proc. IEEE Int'l Communications Conf. (ICC '09), Dresden, Germany, Jun. 2009.

[27] F. Classen and H. Meyr, "Synchronization algorithms for an OFDM system for mobile communication," ITG-Fachtagung, Oct. 1994.

[28] Z. Ye, C. Duan, P. Orlik, and J. Zhang, A Low-Complexity Synchronizer Design for MB-OFDM Ultra-Wideband Systems, MERL Tech. Report Cambridge, USA, Aug. 2007.

[29] K. K. Parhi, VLSI Digital Signal Processing Systems. Hoboken, NJ: John Wiley \& Sons Inc., 1999.

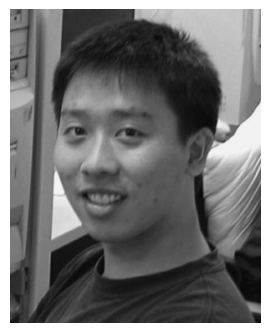

Zhenzhen Ye (S'07-M'10) received the Ph.D. degree in Electrical Engineering from Rensselaer Polytechnic Institute in 2009. He received his B.E. degree from Southeast University, Nanjing, China, in 2000, the M.S. degree in high performance computation from Singapore-MIT Alliance (SMA) program, National University of Singapore, Singapore, in 2003, and his M.S. degree in electrical engineering from University of California, Riverside, CA in 2005.

He is currently an operations research engineer at iBasis, Inc. His research interests lie in the areas of wireless communications and networking, including stochastic control and optimization for wireless networks, cooperative communications in mobile ad hoc networks and wireless sensor networks, and ultra-wideband communications.

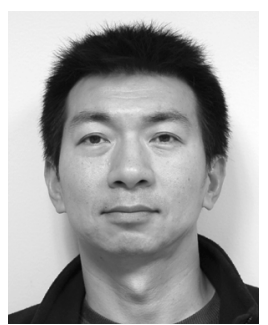

Chunjie Duan received the B.Sc. from Tsinghua University, Beijing, China, M.Sc. from Colorado State University and $\mathrm{PhD}$ from University of Colorado at Boulder, all in Electrical Engineering.

He worked at Alcatel Bell Telephone as a system engineer from 1993 to 1996 . He was with Qualcomm Inc's Infrastructure divsion, which later became Ericsson Wireless Communications, from 1998 to 2004 as a senior engineer in CDMA system and hardware designs. Since 2004, he has been with Mitsubishi Electric Research Labs in Cambridge, Massachusetts, where he is currently a Principal Technical Staff. Dr. Duan's research interests are in physical layer design and signal processing in wireless and optical communications, as well as VLSI implementations.

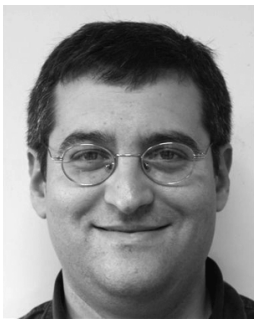

Philip V. Orlik (S'97-M'99) was born in New York, NY in 1972. He received the B.E. degree in 1994 and the M.S. degree in 1997 both from the State University of New York at Stony Brook. In 1999 he earned his Ph.D. in electrical engineering also from SUNY Stony Brook.

He is currently a principal technical staff member at Mitsubishi Electric Research Laboratories Inc. located in Cambridge, MA. His primary research focus is on advanced wireless and mobile cellular communications, sensor networks, ad hoc networking and UWB. Other research interests include vehicular/car-to-car communications, mobility modeling, performance analysis, and queuing theory.

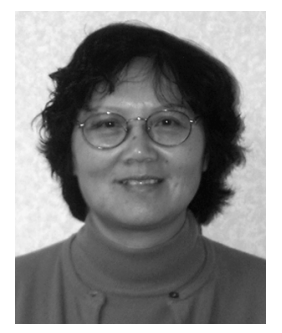

Jinyun Zhang received the B.Sc. degree in radio electronics from Tsinghua University, Beijing, China in 1970. Following her graduation, she was with Tsinghua University until 1985. She received her Ph.D. degree in electrical engineering from University of Ottawa, Canada in 1991.

Dr. Zhang then joined Nortel Networks, where she held various management positions and engineering positions of increasing responsibility in the areas of digital signal processing, wireless communication and optical networks. Since 2001, Dr. Zhang has been the Manager of the Digital Communications \& Networking Group at Mitsubishi Electric Research Laboratories (MERL), Cambridge, MA, USA. Currently, she is leading various new broadband wireless communications and networking research projects that include UWB, ZigBee ad hoc networking, MIMO, broadband multimedia home networking, wireless sensor networks, high speed WLAN, cooperative communications, WiMAX and next generation mobile communications systems.

Dr. Zhang has authored and co-authored more than 130 publications, invented and co-invented more than 100 patents and patent applications, and made numerous contributions to international wireless communications standards. Dr. Zhang is a Fellow of the IEEE and a member of the IEEE AP, BT, COMM, IT, ITS, LEO, SP, and VT Societies. She serves as an Associate Editor of IEEE Transactions on Broadcasting, an AdCom member of IEEE Broadcasting Technology Society, and has served as a Technical Program Committee member for various IEEE conferences.

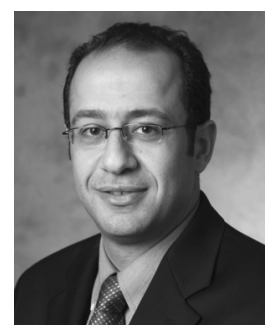

Alhussein A. Abouzeid received the B.S. degree with honors from Cairo University, Cairo, Egypt in 1993, and the M.S. and Ph.D. degrees from University of Washington, Seattle, WA in 1999 and 2001 , respectively, all in electrical engineering.

From 1993 to 1994, he was with the Information Technology Institute, Information and Decision Support Center, The Cabinet of Egypt, where he received a software engineering diploma. From 1994 until 1997, he served as a Project Manager in the Middle East Regional Office of Alcatel telecom, designing integrated voice/data enterprise solutions, and responsible for the technical launch of new Alcatel enterprise and public data switches. During his $\mathrm{PhD}$ study, he held summer appointments with Allied Signal -now HoneywellRedmond, WA, in 1999 and Hughes Research Labs, Malibu, CA, in 2000. In 2001 he joined Rensselaer Polytechnic Institute (RPI), Troy, NY, where he is currently an associate professor in the Electrical, Computer and Systems Engineering Department, and Deputy Director of the Center for Pervasive Computing and Networking, which he co-founded. Since December 2008, he has been Program Director (on leave from RPI), Directorate for Computer \& Information Science \& Engineering, Division of Computer \& Network Systems, National Science Foundation, Arlington, VA, where he is responsible for the Networking Technology and Systems program.

Dr. Abouzeid received the NSF Faculty Early Career Development Award (CAREER) in 2006. His research focuses on protocols for dynamic networks. He serves on various conferences organization committees and is an Area Editor for Elsevier Computer Networks journal. He is an active member of IEEE and ACM. 